Portland State University

PDXScholar

$10-1-1980$

\title{
Cytologic Characterization of Human Constitutive Heterochromatin
}

Timothy Atchison Donlon

Portland State University

Follow this and additional works at: https://pdxscholar.library.pdx.edu/open_access_etds

Part of the Biology Commons, and the Genetics Commons Let us know how access to this document benefits you.

\section{Recommended Citation}

Donlon, Timothy Atchison, "Cytologic Characterization of Human Constitutive Heterochromatin" (1980). Dissertations and Theses. Paper 3092.

https://doi.org/10.15760/etd.3085

This Thesis is brought to you for free and open access. It has been accepted for inclusion in Dissertations and Theses by an authorized administrator of PDXScholar. Please contact us if we can make this document more accessible: pdxscholar@pdx.edu. 
AN ABSTRACT OF THE THESIS OF Timothy Atchison Donlon for the Master of Science in Biology presented December 11, 1979.

Title: Cytologic Characterization of Human Constitutive Heterochromatin.

APPROVED BY MEMBERS OF THE THESIS COMMITTEE:

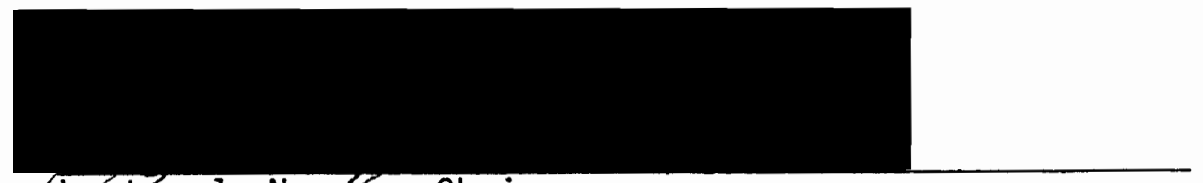

CLester J. Newnan, Chairman

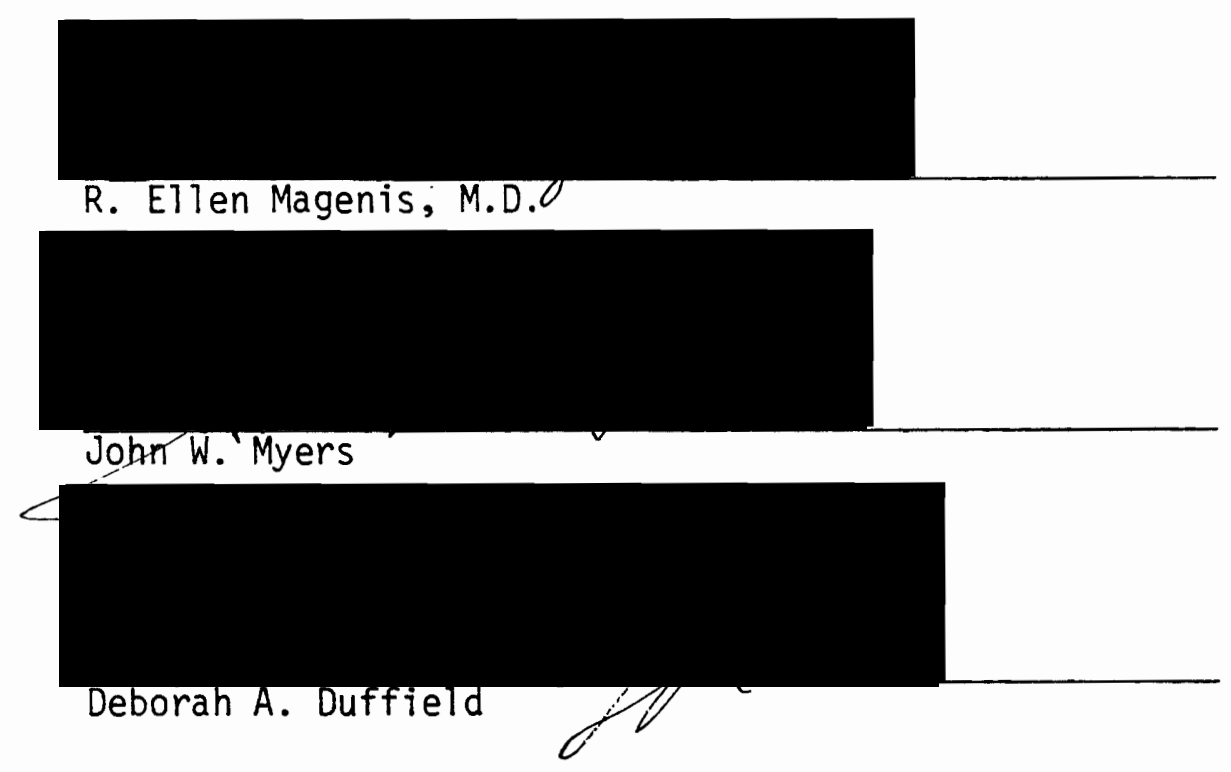

A study was conducted to cytologically map certain subsets of constitutive heterochromatin onto specific portions of human chromosomes. This involved sequentially staining metaphase chromosomes from lymphocytes first with the Centromeric Dot, Giemsa-11, G-banding or Lateral Asymmetry staining techniques, which are cytochemical methods which stain particular chromosomes or chromosomal regions. Then those 
same metaphase chromosomes were stained using the C-banding technique, a method which is believed to denote constitutive heterochromatin. With the exception of the G-banding technique, areas depticted by the special staining techniques were found to reside on]y within the areas stained by the C-band technique and are thus believed to be subsets of constitutive heterochromatin. In addition to portions of the C-band regions, G-banding stained regions throughout the chromosome arms but, only those residing within the $\mathrm{C}$-band regions were considered to be subsets of constitutive heterochromatin. It was found that those regions identified by the Giemsa-11, Centromeric Dot and G-banding techniques were mutually exclusive of one another and occupied discrete regions within the $\mathrm{C}$-bands.

A cytological map was constructed to define the most common locations for these subsets of heterochromatin. The term "common" is used because different individuals in the population may have subtle differences (variants) in the amounts and locations of these subsets. Chromosomes from small families were examined with the various staining techniques and the variants noted. These variants were consistent within different cells of an individual and between related individuals and were inherited in simple co-dominant Mendelian fashion.

Particular chromosomes were found which contained either unusual amounts of $\mathrm{C}$-band heterochromatin or contained this heterochromatin in unusual locations such as those presumably derived by pericentric inversions. C-banding has been used to identify chromosomes having pericentric inversions. Through the use of sequential staining techniques it was discovered that not all of these are actually pericentric inversions. Those chromosomes which contained unusual amounts of heterochromatin were also examined with sequential staining. It was 
found that G-band heterochromatin is more dynamic than Giemsa-11 heterochromatin in the respect that it has undergone more gradual variation in size while differences in Giemsa-11 heterochromatin are less common. Increased amounts of Giemsa-11 heterochromatin certainly exist but are usually expressed as duplications of preexisting blocks of material. Possible mechanisms for these variations, such as unequal crossing over are discussed.

Comparisons of these techniques with Lateral Asymmetry suggest that simple Lateral Asymmetry is common in G-band heterochromatin while compound Lateral Asymmetry is common in Giemsa-11 heterochromatin. Lateral Asymmetry is a property related to the distribution of thymine between the two strands of the double helix. Its implication to chromosome structure and evolution is discussed. 


\title{
CYTOLOGIC CHARACTERIZATION OF HUMAN CONSTITUTIVE HETEROCHROMATIN
}

\author{
by
}

TIMOTHY ATCHISON DONLON

A thesis submitted in partial fulfillment of the reguirements for the degree of

\author{
MASTER OF SCIENCE \\ in \\ BIOLOGY
}

Portiand State University 
TO THE OFFICE OF GRADUATE STUDIES AND RESEARCH:

The members of the Committee approve the thesis of

Timothy A. Donlon presented October 1, 1980.

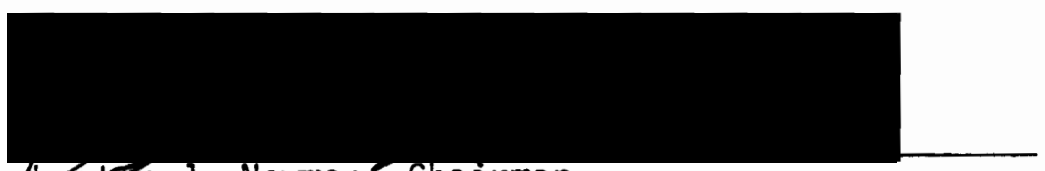

Lester J. Newman, Chairman
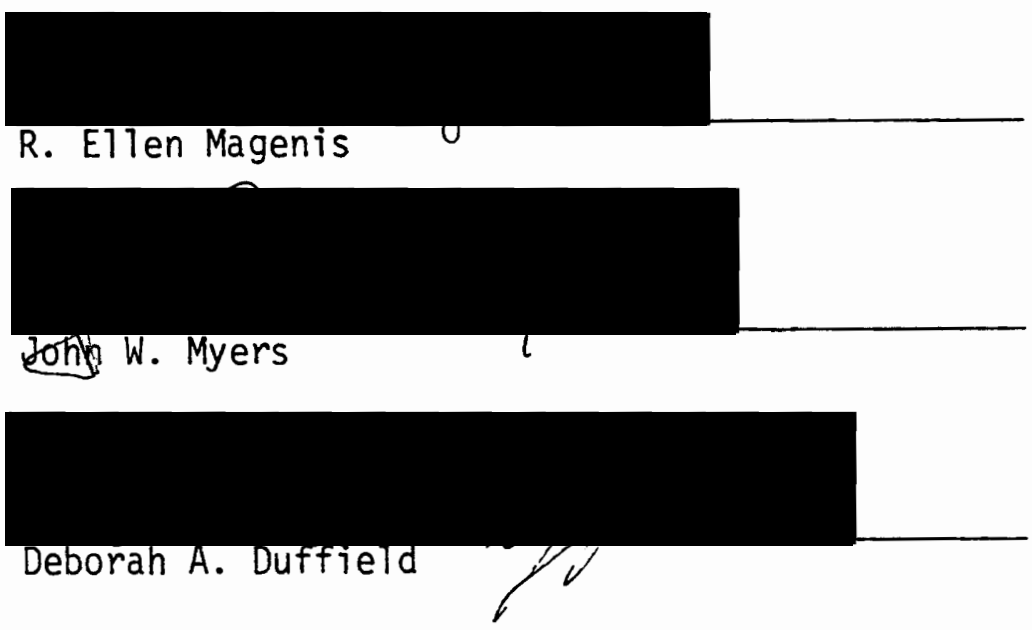

APPROVED:

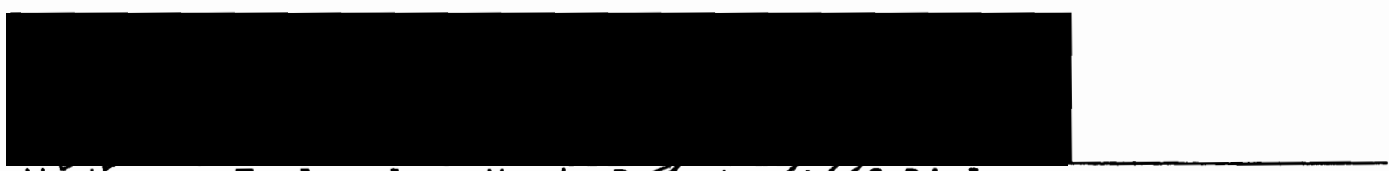

W. Herman Taylor Jr., Head, EgpartmentCof Biology

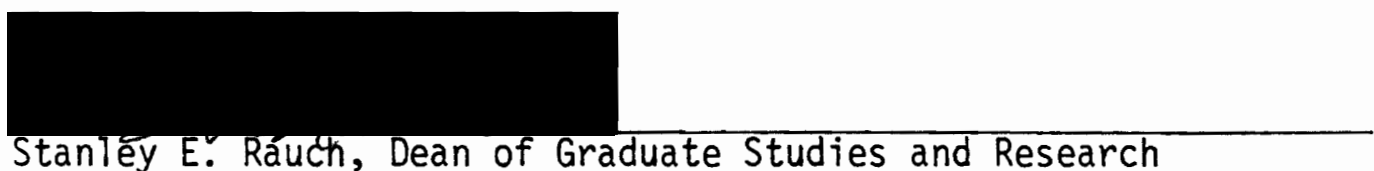

Stanlēy E. Ráuch, Dean of Graduate Studies and Research 


\section{AKNOWLEDGMENTS}

I gratefully aknowledge R.E. Magenis and L. J. Newman for listening to my ideas and permitting me to formulate my questions. Their guidance was essential to the achievement of this study and to my perpetual source of questions. I would also like to extend gratitude toward H. E. Wyandt, D. Duffield and D. Linder for their stimulating conversations which greatly influenced my directions of pursuit. I thank D. Hepburn, M. Brown, C. 01sen, M. Yoshitomi, D. Bangs, S. Bennett and F. Fowler in the Clinical Cytogenetics Laboratory at the University of Oregon Health Sciences Center for screening the chromosomes on many of the patients used in this study and supplying an endless source of excellent chromosome preparations.

Thank you Kathy, for the patience. 
TABLE OF CONTENTS

PAGE

AKNOWLEDGMENTS

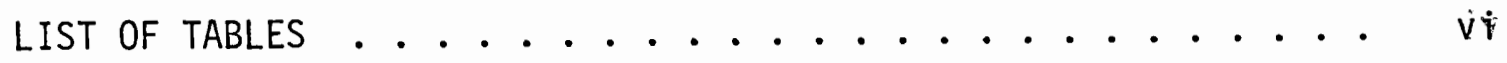

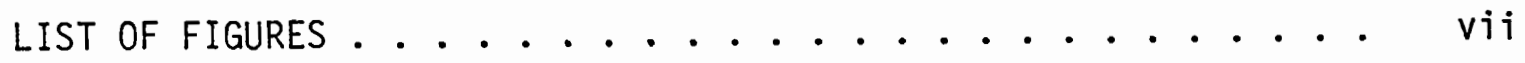

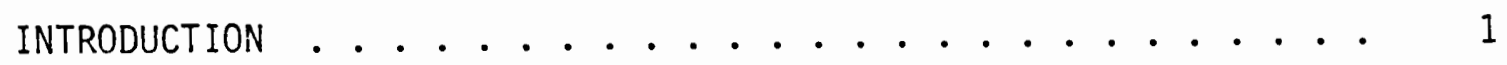

Chromosome Structure ................. 2

Microscopic Analysis . . . . . . . . . . . . . . . . . 2
Biochemical Analysis 2

Visualization of Constitutive Heterochromatin . . . . . 7

C-banding ................... 7

G-banding ................. 8

Giemsa-11 Staining .............. . . 9

Centromeric Dot Staining .......... . . 10

Lateral Asymmetry ............... 11

Objectives .............................. 13

MATERIALS AND METHODS............................. 15

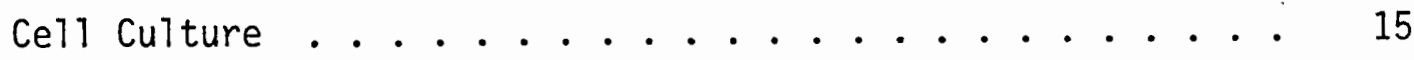

Slide Preparation......................... 16

G-banding . . . . . . . . . . . . 16

Giemsa-11 Staining ............... 16

C-banding ...................... 17

Lateral Asymmetry . . . . . . . . . . . 18

Centromeric Dot Staining ............ 18

Photography..................... 19

RESULTS ........................... 20

General Description of Chromosomes ......... 23 
PAGE

Chromosome 1... . . . . . . . . . . . . . 23

Chromosomes $2-5$. . . . . . . . . . . . . 25

Chromosomes 6-9 \& $\mathrm{X}$. . . . . . . . . . . . . . 25

Chromosomes $10-15,21 \& 22$. . . . . . . . . . . . . 27

Chromosomes 16-20 \& $Y$. . . . . . . . . . . . . 28

Lateral Asymmetry . . . . . . . . . . . . . . 32

Variations in C-band Size and Organization . . . . . . . 36

Other Chromosome Variants . . . . . . . . . . . . 42

DISCUSSION . . . . . . . . . . . . . . . . . . . . . . 48

Possible Mechanisms of Chromosome Variation . . . . . . . 52

Meiotic Origin . . . . . . . . . . . . . 52

Mitotic Origin ................. 54

Gene Amplification............ . . . 54 


\section{LIST OF TABLES}

TABLE

PAGE

I Satellite DNAs and their Chromosome Locations . . . . 6

II Satellite DNA Content and Chromosomal Staining ..... 49 


\section{LIST OF FIGURES}

FIGURE

PAGE

1. Banding of a Variant Chromosome 6 . . . . . . . . 22

2. Idiograms of Chromosomes 1-5 . . . . . . . . . . . 24

3. Idiograms of Chromosomes 6-12 \& X . . . . . . . . . 26

4. Idiograms of Chromosomes $13-22 \& Y$. . . . . . . . . . 29

5. Karyotype Demonstrating Lateral Asymmetry . . . . . . . 31

6. Chromosome 9 with Giemsa-11, C-banding and Lateral

Asymmetry .................. 33

7. Chromosome 1 with Lateral Asymmetry, Giemsa-11 and

C-banding . . . . . . . . . . . . . . 35

8. Chromosome 1 Variants with Giemsa-11 and C-banding . . . 37

9. Heretability of Chromosome 1 with Giemsa-11 and

C-banding . . . . . . . . . . . . . . .

10. Chromosome 9 Variants with G-banding, Giemsa-11 and

C-banding . . . . . . . . . . . . . .

11. Variant Chromosomes 1,3,4 \& 5 with G-banding, Giemsa-11 and C-banding . . . . . . . . . . . . . .

12. Variant Chromosomes $6,9,11,13,14$ \& 15 with G-banding,

Giemsa-11 and C-banding .............

13. Variant Chromosomes $16,17,18,19,20,21$ \& 22 with G-banding,

Giemsa-11 and C-banding . . . . . . . . . . 


\section{INTRODUCTION}

Deoxyribonucleic acid (DNA) contains the genetic information necessary for cell proliferation and the differentiation of all life as we now conceive it. This DNA is generally found in intimate association with certain proteins to produce a more compact structure called chromatin. During growth and cell division this genetic material must be replicated and divided into two equal amounts so that each new daughter cell receives a full complement of genetic information (genome). To accomplish this neatly and without undue complication the chromatin must further be compacted into structures which are more readily maneuvered. This involves a further association of the chromatin with still more proteins to form what are known as chromosomes. Humans contain 23 pairs of different chromosomes and the morphology of these chromosomes is consistent not only from cell division to cell division but also from generation to generation. It has long been known that the DNA in specific portions of chromosomes (euchromatin) is transcribed into messenger RNA which will be used by the cell to eventually promote growth and sustain life. Other portions of the chromosomes contain DNA which are transcriptiona1ly inert (Brown,1966) and serve no known function. These poorly understood portions of each chromosome are known as constitutive heterochromatin and will be the focal point of this study with the expectancy that some understanding will be gained as to why it exists. 
Constitutive heterochromatin should not be confused with "facultative" heterochromatin which is the innactivated euchromatin of one of the $x$ chromosomes of most normal female mammals. It is known that all mammals have one functionally active $X$ chromosome whether they be male or female and that in the female, who generally has two present, there is innactivation of one X chromosome (Lyons, 1961). Individuals having more than two $X$ chromosomes will have all but one of them innactivated. Although the actual mechanism of innactivation is not clearly understood it is accomplished by condensation of the genetic material on this chromosome to yield a compact body (Barr body) which is visible throughout the cell cycle (Barr, 1949). Because this type of heterochromatin is regulated and clearly different than constitutive heterochromatin it will be excluded from any further reference to heterochromatin.

As a matter of historical interest heterochromatin was first cytologically described in the moss Pellia epiphylla in 1928 by the German geneticist Emil Heitz. He suggested the term euchromatin for those portions of the chromosomes which become invisible in late telophase and heterochromatin for those which remain condensed throughout the entire cell cycle.

\section{Chromosome Structure}

To gain an understanding of what heterochromatin is will necessitate a brief description of chromosome structure as revealed by light and electron microscopy and some biochemical studies performed on the DNAs which make up the heterochromatic regions.

Microscopic Analysis. With the aid of the light microscope chromosomes appear as two parallel rods of equal length held together 
at a point called the primary constriction or centromere. In humans the centromere may be located near one end of the rods, in the middle or to one side of the middle to produce acrocentric, metacentric or submetacentric chromosomes, respectively. The portions on either side of the centromere are called arms and those that are smaller in acrocentric and submetacentric chromosomes are referred to as short arms while the larger arms are called long arms. The true metacentric chromosome has two arms of equal length. All human chromosomes contain some constitutive heterochromatin surrounding the centromere and chromosomes 1,9 and 16 have a. substantial amount which is located predominantly in the long arms. While chromosome 1 is metacentric the heterochromatin is on the arbitrarily designated long arm.

DNA measurements using ultraviolet light absorption and Feulgen stained metaphase preparations reveal that the heterochromatic region contains more DNA per unit area than the adjacent euchromatic chromosome arms (Geraedts et a1.,1975). Thus it can be said that these regions are more compact or dense in relation to euchromatin. Variations in lengths of these regions are accompanied by variations in DNA content (Geraedts et a1.,1975). This suggests that increases in the length of the heterochromatic regions correspond to an actual increase in DNA content.

Electron microscopy of metaphase chromosomes has revealed some interesting information (see Comings,1978, for review). The basic structure of chromatin is very similar throughout the metaphase chromosome whether it is euchromatin or heterochromatin. The $25 \AA$ thick double helix of DNA is wound around an octamer, made up from four histone 
proteins, to form a $100 \AA$ thick fiber with a beaded appearance. These beads are referred to as nu bodies or nucleosomes. This fiber is once again coiled into a solenoid structure with a thickness of 250$300 \AA$. To this point chromatin from all portions of the chromosome are very similar. Additional packaging of the chromatin into the chromosome requires a large group of nonhistone proteins and involves not the further coiling of these fibers but the gathering of them into what have been called "radial loops" by Marsden and Laemmli (1979) or "rosettes" by Okada and Comings (1979). The average rosette is composed of $20.7 \pm 5.3$ radial loops and contains 13.7 um \pm 4.8 um of naked DNA in histone depleted samples. The naturally occuring rosette would of course contain a much shorter segment of whole, native chromatin (containing histones). These rosettes are linked together by a segment of DNA 4.2 um \pm 1.7 um long (also measured as naked DN.4). The loops are held together in the rosette by what have been termed "nuclear matrix proteins" by Comings and Okada (1976) and "scaffolding proteins" by Paulson and Laemmli (1977). These proteins comprise a very heterogeneous class of chromosomal proteins. At least some are involved with directly linking the rosette cores to one another possibly for structural support, while others are involved with duties such as DNA repair and transcription. The loops or rosettes do not assume an even distribution throughout the length of the chromosome but form clusters which correspond well in size and location to dense bands of chromatin (observed in many organisms with the light microscope) called chromomeres (Okada and Comings, 1974). The heterochromatic regions of chromosomes contain a higher density of rosettes than the surrounding areas (Okada and Comings, 1979). 
It seems that one of the major differences between euchromatin and heterochromatin is the density of chromatin. The types of nonhistone proteins which are highly heterogeneous are also likely to be different in distinct portions of a chromosome.

Biochemical Analysis. Neutral density ultracentrifugation of whole human DNA reveals the presence of several peaks or "satellites" which separate from the main bulk of DNA (see Macaya et a1.,1977, for review). The majority of these satellites are light relative to the main bulk of DNA and AT rich and are designated satellites I,II,III and IV. Heavy (GC rich) satellites also exist (e.g. satellites A,B,C and D; see Table I for comparisons). Most of these satellite DNAs have also been isolated by reannealing studies and are known to contain highly repetitive base sequences (Marx et a1.,1976). Using in situ hybridization (which involves making a radioactive DNA probe and annealing it to complementary sequences on denatured, single stranded metaphase chromosomes on slides it is possible to locate these repetitive DNAs on regions of specific chromosomes (Gosden et a1.,1975b). As shown in Table I most of these satellite DNAs are localized to the centromeric portions of the chromosomes (corresponding to the heterochromatic portions). Strand separation and base analys is of the AT rich components reveals a difference in the distribution of thymine between the two complementary strands of the DNA duplex (Evans,1976). This characteristic will be dealt with in more detail below.

In summary the heterochromatin of humans is very condensed, AT rich and composed of highly repetitive DNA sequences. 
SATELLITE DNAS AND THEIR CHROMOSOMAL LOCATIONS

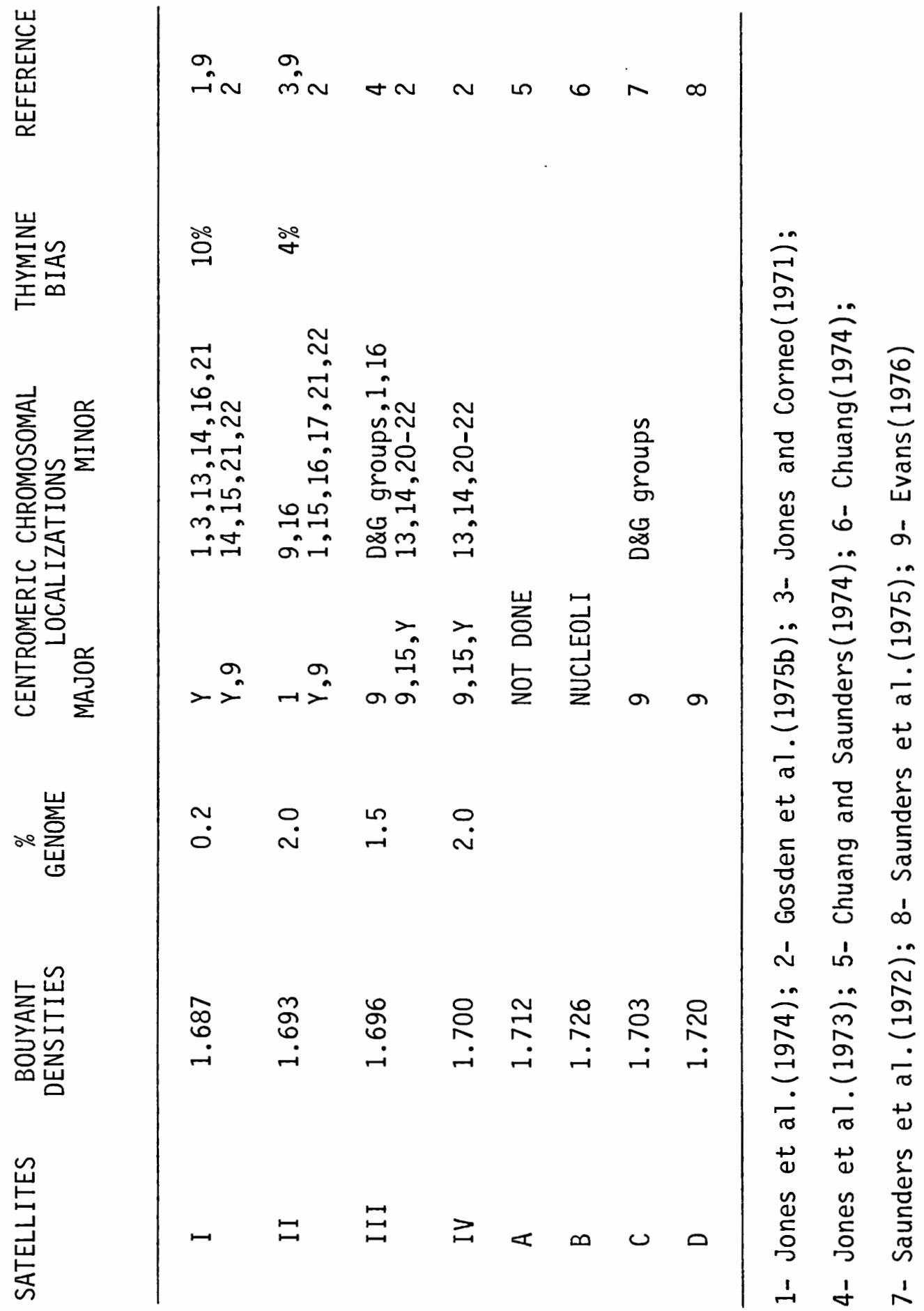


Visualization of Constitutive Heterochromatin

Constitutive heterochromatin may be visualized in metaphase chromosomes by a technique referred to as C-banding and shows such profound variations in size and amount that it may be considered to be virtually unique for a given individual (Müller et a1.,1975). Certain special staining techniques have become available which permit the identification of specific subsets of the C-band heterochromatin: (G-banding, Seabright,1971; Giemsa-11, Bobrow et a1.,1972, Gagne' and Laberge,1972; and Lateral Asymmetry, Angell and Jacobs,1975). These techniques have not previously been compared to each other so it is not clear whether or not several techniques are staining the same or cytologically discrete regions of constitutive heterochromatin. It is the purpose of the present microscopic study to examine the substructural organization of such regions to supply valuable information on the composition, variability and production of specific heterochromatic regions.

C-banding. Constitutive heterochromatin may be directly visualezed in human metaphase chromosomes by a technique referred to as C-banding (Arrighi and Hsu,1971). The differential staining produced by this technique is believed to be a result of defferential extraction of DNA from the chromosome (Comings et al.,1973). A more recent study by Holmquist (1979) has been published which measures the parameters involved in C-banding. His study shows that C-banding involves the differential solubilization of fragmented DNA from euchromatin by depurination, DNA denaturation and chain breakage at the depurinated sites, in that order. Depurination is accomplished by treating the metaphase preparation with $0.2 \mathrm{~N} \mathrm{HCl}$ for $10 \mathrm{~min}$. although some depurination may occur in the acid/methanol fixation step. C-banding also 
involves the use of a mild concentration $(0.01-0.07 \mathrm{~N}$ ) of $\mathrm{NaOH}$ which serves to reduce the integrity of the DNA molecule so that it may be broken at the apurinic sites, solubilized and extracted in a hot salt treatment. Because heterochromatin is more condensed than euchromatin, a result of having more nonhistone protein and DNA, the DNA in heterochromatin is less accessible to the destructive forces applied and therefore is more resistent to solubilization. The resulting chromosomes contain more DNA in the heterochromatin than in the euchromatin and subsequently bind disproportionate amounts of Giemsa dye.

G-banding. Chromosome preparations subjected to a similar treatment as the C-banding technique under milder conditions such as shorter exposure to $\mathrm{NaOH}$ will reveal not only $\mathrm{C}$-bands but other bands as well, called Giemsa bands or-G-bands. These G-bands correspond to the naturally occurring chromomere pattern (Okada and Comings, 1974) and are related to DNA density differences as revealed by Feulgen staining (Rodman,1974). Other methods which suggest these bands to contain more chromatin than the negative staining regions are phase microscopy (McKay,1973) and electron microscopy (Bahr et al.,1973). The mechanism for producing G-bands on acid/methanol fixed chromosomes by $\mathrm{NaOH}$ and hot salt are probably very similar to those for C-banding. DNA is solubilized and removed from the chromosome in those regions which are less condensed (the pale or interbands) while the more condensed regions (G-bands) are less likely to be solubitized arid removed. These more condensed regions are then able to bind a proportionately greater amount of Giemsa stain (Comings and Avelino,1975) to reveal longitudinal striations or G-bands. 
G-banding may also be achieved by digesting chromosomes with proteolytic enzymes such as trypsin followed by Giemsa staining. Presumably the trypsin treatment cleaves proteins (possibly nuclear matrix proteins) which disperses the chromatin slightly to increase Giemsa binding in those regions (chromomeres) which were inaccessible to dye before because of their state of increased condensation (Comings, 1978). The trypsin G-banding technique does not stain all the constitutive heterochromatin. Chromosome 9 contains a moderate size of C-band heterochromatin but remains relatively unstained in this region with G-banding. Because nonhistone proteins have been found to be associated with constitutive heterochromatin (Matsukuma and Utakoji,1976) Comings (1978) believes that some of them mask the DNA of certain regions to prevent Giemsa from binding. It may well be that certain chromosomal proteins are more resistant to trypsin hydrolys is than others. Merrick et al. (1973) have shown that prolonged digestion with trypsin does eventually lead to chromosomes identical to those produced by the C-banding technique. It is this differential staining in different heterochromatic regions which will be of importance in this study. Because G-banding also stains regions other than $\mathrm{C}$-band heterochromatin sequential staining from G-banding to $\mathrm{C}$-banding will be performed on the same metaphase preparations to accurately delineate which G-bands reside within the C-band regions.

Giemsa-11 Staining. Another method which differentiates subsets of constitutive heterochromatin is the Giemsa-11 technique (Bobrow et al., 1972; Gagné and Laberge,1972) which utilyzes Giemsa under alkal ine conditions for the differentiation of certain C-band regions. The chromosomes for the most part appear pale blue while certain C-bands 
or portions of $\mathrm{C}$-bands stain intensely red. The mechanism to this seemingly complicated reaction is poorly understood but Wyandt et al. (1976) have identified the two active ingredients in Giemsa responsible for the staining (Azure $B$ and Eosin $Y$ ). They believe that removal of certain acidic chromosomal proteins (nonhistone) under the alkaline conditions ( $\mathrm{pH}$ 11.3-11.6) facilitates the precipitation of a red Azure/Eosin complex onto these regions (personal communication). That this technique differentiates a subset of heterochromatin is evidenced by the fact that not all human chromosomes stain with Giemsa-11 but that all of them contain C-band heterochromatin. One objective of this study is to determine which chromosomes stain with Giemsa-11 and to accurately determine its intra-chromosomal location.

Centromeric Dot Staining (Cd). The centromeric dot (Cd) technique was first described in humans by Hans Eiberg in 1974. It was referred to as centromeric dot staining because each chromosome had a pair of dots at the primary constriction suggesting that the centromeres were staining. Known dicentric chromosomes were later shown to contain two pairs of dots supporting this idea (Marks,1977). The technique involves subjecting the chromosome preparations to fairly harsh treatment (45 min. in isotonic saline adjusted to $\mathrm{pH} 8.5$ at $85^{\circ} \mathrm{C}$ ) and for this reason is thought to be similar to the C-banding technique in its extreme sense in that all but the most condensed or resistant chromatin is removed. In fact if one over treats preparations in $\mathrm{NaOH}$ in the C-band technique Cd stained chromosomes are often produced.

The unequal longitudinal distribution of chromatin in fixed metaphase preparations seems to be the major basis for many of the Giemsa related banding techniques such as those employed throughout this study. 
The differential staining is facilitated by the selective dispersion of chromatin in certain chromosomal regions by the removal of protein (Comings and Avelino,1975) by selective solubilization and removal of DNA (Mittermayer et al.,1971) or both of these mechanisms to varying degrees.

One of the objectives of this study was to perform the Cd technique on a type of chromosome variant which was reported by Madan and Bruinsma (1979) in which chromosomes 6 often reveal heterogeneity in a light staining region at the primary constriction when examined with G-banding. The variation in G-banding may reflect differences in amount of centromeric material. Using longer chromosomes it is becoming apparent that all chromosomes have some light staining material at the primary constriction when stained for G-banding (see Yunis,1976).

Lateral Asymmetry. The term "lateral asymmetry" refers to a staining technique which permits one to indirectly visualize the unequal distribution of the deoxyribonucleotide thymine within the DNA molecule which makes up a given chromatid. It is currently accepted that one DNA molecule (double helix) runs the entire longitudinal axis of a single chromatid and that two sister chromatids compose a given chromosome in the typical human somatic metaphase. As the DNA molecules (chromatids) were replicated semi-conservatively, each containing half of the original double helix from the parental chromatid, the two chromatids should be virtually identical with regards to their total base pair constitution. This offers one the chance to examine the distribution of bases within a single double helix DNA molecule and to visualize differences on a chromosomal level. Human constitutive heterochromatin is known to be AT rich but these two bases are not 
present in the other half of the DNA molecule with equal frequencies. For example, in Table I it can be seen that satellites I and II have been found to contain $10 \%$ and $4 \%$ differences respectively in the distribution of thymine between the two strands of the double helix. Thus cells grown for one replication in the thymidine analogue 5-bromodeoxyuridine (BrdU) show an asymmetrical incorporation into the daughter DNA duplexes (chromatids)(Latt et a1.,1974).

---AAAGCAAAGCAAAGCAAAGC---

---TTTCGTTTCGTTTCGTTTCG--,

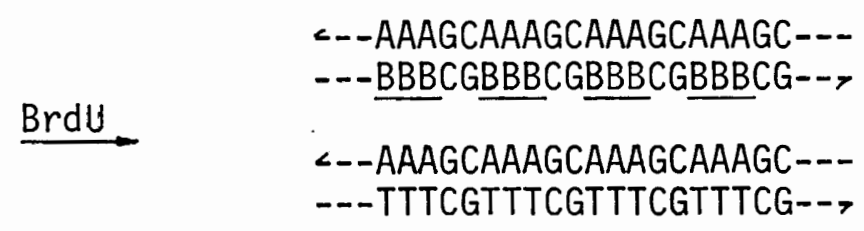

The resultant differences may be visualized microscopically at the chromosomal level (Lin et al.,1974). Those regions of the chromatid which incorporate more BrdU stain less intensely than those same regions on the sister chromatid. The example presented above contains a consistent orientation of its thymine bias. This will produce a chromosome in which one entire chromatid will label (stain less intensely) within the C-band region. This type of asymmetry is referred to as "simple lateral asymmetry" (Angell and Jacobs,1975). In some cases the thymine bias may "switch" from one strand to the other within the heterochromatic region as shown below.

--AAAGCAAAGCGCTTTGCTTT----. TTTCGTTTCGCGAAACGAAA--,

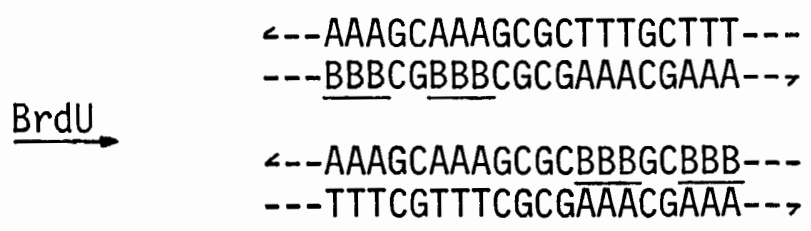

The resultant chromosome will appear with alternating staining, a portion of one chromatid dark, that same portion on the sister chromatid light and the remaining portion will appear light on the prior chromatid and dark on that same portion of the sister chromatid. 
This more complex form is referred to as "compound lateral asymmetry". Different chromosomes demonstrate different types of lateral asymmetry. Simple lateral asymmetry reportedly exists on many chromosomes 1 and more commonly on 15,16 and the $Y$ (Angell and Jacobs,1975). Compound lateral asymmetry has been documented on chromosome 1 (Angell and Jacobs, 1975). Lin and Alfi (1978) have found asymmetry on most human chromosomes. It may be so complex on chromosomes 9 that both chromatids appear to stain symmetrically dark. Galloway and Evans (1975) believe that this condition results from the thymine bias "switching" back and forth so many times as to escape detection by cytologic means.

\section{Objectives}

The objectives of this study were to compare the distribution of constitutive heterochromatin in chromosome complements (karyotypes) from various individuals using the five staining techniques; C-banding, G-banding, Giemsa-11, Cd and lateral asymmetry. The following questions will be adressed:

1) What chromosomal regions stain with each technique? This knowledge may help to identify small derivative chromosomes which are now too small to be deciphered by conventional techniques.

2) Does the material stained by one technique occupy the same site as that stained by another or are they cytologically discrete? This will help to categorize different "types" of heterochromatin.

3) What sort of "normal" variation can one expect in these heterochromatic regions with a particular staining technique? From a clinical standpoint this will be of value in knowing how to interpret "normal" from "abnormal" chromosome complements. 
4) Is the organization of those regions which stain consistent within an individual and heritable within a family? New chromosome "markers" are always being sought for help to map genes onto chromosomes by relating the segregation of these chromosomes in a family with the segregation of known genes and gene products.

5) Will the details in organization of these heterochromatic regions, furnished by the staining techniques, add support to current mechanisms postulated to account for the formation of heterochromatically variant chromosomes? The mechanisms postulated to account for size variations in heterochromatin include unequal crossing over, unequal sister chromatid exchange and over-replication of certain sequences of DNA. This study could furnish support for certain of the postulated mechanisms.

6) Does a given type of lateral asymmetry occur predominantly in certain types of heterochromatin? This will help to answer whether changes in thymine bias occur between cytologically discrete types of heterochromatin and whether thymine biases may occur within a given type of heterochromatin. 
MATERIALS AND METHODS

Cell Culture

Peripheral blood samples from 1,850 individuals were obtained, heparin added to prevent coagulation and the samples left to settle for 1-3 hrs. in order to facilitate separation of the red blood cells and to allow removal of the fymphocyte containing "buffy coat". From one half to $1 \mathrm{ml}$. of this serum/lymphocyte suspension was added to a tissue culture flask containing $10 \mathrm{mls}$. of RPMI 1640 medium (GIBCO) supplemented with fetal calf serum (10\%). Cell division was stimulated with the mitogen phytohemagglutinin at a concentration of $90 \mathrm{ug} . / \mathrm{ml}$. and the cells left to grow at $37^{\circ} \mathrm{C}$ for $66 \mathrm{hrs}$. after which time colchicine was added at a final concentration of $0.05 \mathrm{ug} . / \mathrm{ml}$. to arrest mitoses. Harvesting of cells took place as follows. After $2 \mathrm{hrs.}$. in colchicine the cells were centrifuged at $1,000 \mathrm{r.p.m}$. $(183 \times \mathrm{g})$ for $6 \mathrm{~min}$., washed in $5 \mathrm{mls}$. of Hanks' balanced salt solution (GIBCO), spun again, washed twice more with $3 \mathrm{mls}$. of $0.075 \mathrm{M} \mathrm{KCl}$ hypotonic solution and fixed in several changes of methanol/acetic acid (3:1) for a duration of $20 \mathrm{~min}$. each.

Additional cultures were set up for lateral asymmetry. These cells were grown for $48 \mathrm{hrs}$. in medium containing 100 uM 5-bromodeoxyuridine (Sigma), 0.4 uM 5-flourodeoxyuridine and 6 uM uridine (these latter two compounds reduce the toxic effects of $\mathrm{BrdU}$; Lin et al.,1974). The cultures were grown in the dark to prevent photolysis of the substituted DNA. Harvesting of these cultures proceeded as previously described. 


\section{S1ide Preparation}

Cel1 suspensions were dituted in fresh fixative to a final concentration which produced a thin milky suspension and applied dropwise to a clean, wet slide from a distance of about $2 \mathrm{ft}$. The slides were immediately flooded with fresh fixative and allowed to dry.

\section{G-banding}

The procedure used for G-banding was that of Seabright (1971). Slides were dipped in $0.025 \%(\mathrm{w} / \mathrm{v}$ ) tryps in (dissolved in $0.9 \% \mathrm{NaCl}$ ) for 10-30 sec. followed immediately by two rinses of $0.9 \% \mathrm{NaCl}$. After shaking off excess liquid the slides were stained in a horizontal position with $5 \mathrm{mls}$. of Wright's stain at a final concentration of $0.6 \mathrm{mg} . / \mathrm{ml}$. for $2 \mathrm{~min}$. Banding was optimal when tryps in treatment was long enough to produce symmetrical striations in the chromosomes but not so long that they appeared swolien and pale.

\section{Giemsa-11}

The Giemsa-11 technique used for this study was a modification of the method first used by Bobrow et al. (1972) and Gagné and Laberge (1972) in which the two active components of Giemsa (Azure B and Eosin Y) are combined at alkaline pH (Wyandt et al.,1976). A volume of $100 \mathrm{~m} 1 \mathrm{~s}$. of

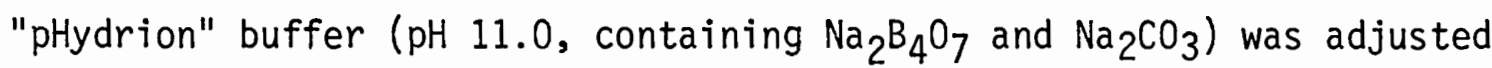
to $\mathrm{pH}$ 11.3-11.6 with concentrated $\mathrm{NaOH}$. This solution was distributed to two $50 \mathrm{ml}$. Coplin jars and warmed in a water bath to $37^{\circ} \mathrm{C}$. To this solution was added $0.6 \mathrm{ml}$. of Azure $B$ and $0.5 \mathrm{ml}$. of Eosin $\gamma^{*}$. Slides between one day six months old were immediately incubated in this buffer/stain for 3-6 min., rinsed in distilled water and blown dry with compressed air. Chromosomes uniformly pale blue were considered 
understained whereas those uniformly pink were considered overstained. Optimally they appeared pale blue with deep red staining at the centromeric areas on many chromosomes, notably on chromosomes 9 and the $D$ and $G$ group chromosomes. The total staining time varied somewhat depending on the age of the slide and the cell density. Time to achieve optimum staining was increased (to over $5 \mathrm{~min}$.) on slides which were either over one month old or contained an unusually large number of cells.

C-banding

It was of great interest to learn how Giemsa-11 and G-band heterochromatin related to the entire block of C-band heterochromatin in it's locations. To reveal C-band heterochromatin on those same metaphase preparations which had previously been G-banded required that the slides be soaked in three changes of fresh xylene to remove immersion $0 i 1$ and destained in $95 \% \mathrm{EtOH}$ for $10 \mathrm{~min}$. Slides were dipped for $5 \mathrm{~min}$. in a 1:6 dilution of $0.07 \mathrm{~N} \mathrm{NaOH}$ in 2XSSC $(0.3 \mathrm{M} \mathrm{NaCl}, 0.03 \mathrm{M} \mathrm{Na}$ Citrate adjusted to $\mathrm{pH} 7.0$ ) rinsed in 2XSSC for $30 \mathrm{sec}$, rinsed in $70 \% \mathrm{EtOH}$ for $30 \mathrm{sec}$, rinsed in another change of $70 \% \mathrm{EtOH}$ for $5 \mathrm{~min}$. followed by several changes of $95 \%$ EtOH. Slides were then blown dry, 5 drops of 2XSSC and a coverslip applied and incubated at $65^{\circ} \mathrm{C}$ overnight (16 hrs.). The next day the slides were cooled and rinsed in two changes each of $70 \%$ and $95 \% \mathrm{EtOH}$ ( $5 \mathrm{~min}$. each) dried and stained $8 \mathrm{~min}$. in $4 \%$ Giemsa/ Gurr's buffer.

*The total dye content of the dry stock samples of Azure B and Eosin $Y$ may vary considerably between different brands and even between different lots of a given brand. Good results were obtained with MC/B Azure B (total dye content 78\%) and Harleco Eosin Y (total dye content $84 \%$ ) employing a 10 fold larger molar concentration of Azure to Eosin. Different total dye contents in different brands will likely require modifications of the stock solutions. 
To produce C-bands on those preparations which had been previousty Giemsa-11 stained the slides were destained in 95\% EtOH and incubated at $65^{\circ} \mathrm{C}$ in 2XSSC overnight as in the method used for G-banded preparations. The remaining steps were the same as those reported in that section.

\section{Lateral Asymmetry}

Chromosome preparations examined for lateral asymmetry were stained according to the technique of Angell and Jacobs (1975). Slides of cells grown in BrdU were stained in the photosensitive dye 33258 Hoechst in distilled water $(0.5 \mathrm{ug} . / \mathrm{ml}$.$) for 15 \mathrm{~min}$. Distilled water was placed on the slide and a coverslip applied and ringed with rubber cement to prevent evaporation. The slides were then exposed to a $200 \mathrm{~W}$ mercury vapor light source at a distance of $38 \mathrm{~cm}$. for $60 \mathrm{sec}$. The coverslips were removed and the slides incubated in a Coplin jar containing 2 XSSC $\left(\mathrm{pH} \mathrm{7.0)}\right.$ at $65^{\circ} \mathrm{C}$ for $1 \mathrm{hr}$., rinsed in distilled water and stained 5 min. in 2\% Giemsa/Gurr's buffer. Metaphase preparations which did not receive a long enough period of $u . v$. light were purple while those receiving too much were uniformly pale blue with the C-band region being poorly differentiated. Optimally the euchromatic portions were pale blue while the centromeric heterochromatin displayed marked contrast between the two sister chromatids; one region stained purple while the adjacent region on the sister chromatid stained a very pale blue or not at al1.

\section{Centromeric Dot Staining}

The Cd technique employed was essentially that originally described by Eiberg (1974) in which one week old slides were incubated for $45 \mathrm{~min}$. 
in Earle's balanced salt solution adjusted to $\mathrm{pH} 8.5$ at $85 \mathrm{C}$. The slides were cooled, rinsed in distilled water and stained 20 min. in $4 \%$ Giemsa/Gurr's buffer.

\section{Photography}

A11 lateral asymmetry, G- and C-banded preparations were photographed on a Zeiss Photoscope II using Kodak High Contrast Copy film and developed in "Diafine" film developer. Giemsa-11 stained slides were photographed on the same microscope using Kodak Pan-X film and developed in "Diafine". 


\section{RESULTS}

Upon examination of the G-banding patterns of the chromosomes in the human karyotype it becomes apparent that all of the chromosomes contain some juxtacentromeric G-band positive heterochromatin, possibly with the exception of the $Y$ chromosome. This is ascertained by directly comparing the G-banded with the C-banded chromosomes using sequential staining. In the typical metaphase the blocks of G-band positive heterochromatin are located on both arms of the chromosomes extending right up to the primary constriction. In studying longer prometaphase chromosomes however, it appears that there are relatively small G-band negative or light regions directly at the primary constrictions within the G-band positive heterochromatin and which seems to correspond to the actual centromeres. Occasionally, chromosomes revealed different amounts of this material and indeed the homologous chromosomes of a given individual may contain size heteromorphisms within this region. For example, chromosome 6 has been reported to demonstrate differences in the amount of this G-band negative material (Madan,1979). C-banding of variant $6 \mathrm{~s}$ in this study reveals this material to be constitutive heterochromatin because, it stains positive with C-banding. Examination of these chromosomes 6 with the Cdot (Cd) technique, which is believed to demark the actual centromeres, has led to some interesting results. This technique is referred to as centromeric dot staining because on most chromosomes there are two dots present, one on each chromatid. The chromosomes 6 examined in this study 
demonstrated at least two sets of dots strongly suggesting the presence of at least two sets of centromeres. Furthermore these dots correlated well in size and location with the G-band negative regions at the primary constrictions (Figure 1). Other chromosomes, such as 1, 9 and 16, occasionally revealed more than one set of dots in this study, as well suggesting that this phenomenon of "duplicated" centromeres is not restricted to chromosome 6 . The evidence supports the idea that there is G-band positive heterochromatin surrounding the primary constriction but the actual centromere is G-band negative. Both types are stained by C-banding and are therefore, by definition, constitutive heterochromatin.

It is clear from this study that there are other portions of C-band heterochromatin that are G-band negative but which do not stain with the Cd technique. These regions are found on chromosomes which contain Giemsa-11 positive heterochromatin and correspond in size and location to those regions stained by Giemsa-11. For example, chromosome 9 contains the largest amount of G-band negative or light heterochromatin and the largest amount of Giemsa-11 heterochromatin.

One conclusion drawn from this study is that C-band heterochromatin may be classified into at least three non-overlapping categories and they are cytologically discrete entities. These classifications are: Cd positive - that which stains with Cd but does not with either G-banding or Giemsa-11. G-band positive - that which stains with G-banding but not with either Cd or Giemsa-11. 


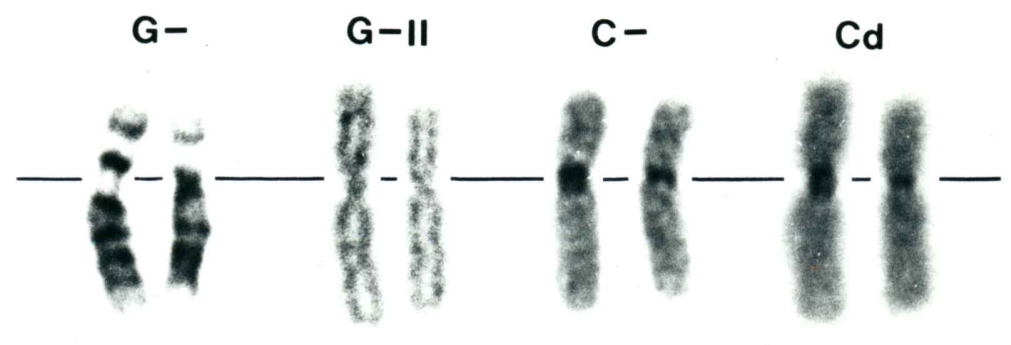

Figure 1. G-banding, Giemsa-11, C-banding and Cd staining of chromosomes 6 from an individual with a marked difference in the centromeric G-band negative heterochromatin. Note the two bands or pairs of dots stained by the $\mathrm{Cd}$ technique. 
Giemsa-11 positive - that which stains with Giemsa-11 but not with either Cd or G-banding.

All three types are stained by C-banding and are therefore by definition constitutive heterochromatin.

This study shows that the lateral asymmetry technique includes all of the above types of heterochromatin with the possible exception of $\mathrm{Cd}$ positive heterochromatin.

\section{General Description of Chromosomes}

Although Cd stained chromosomes are not included in the following figures it can be assumed that all chromosomes contain a small area at the primary constriction or centromere which stain by Cd but are G-band and Giemsa-11 negative.

Chromosome 1. Giemsa-11 and G-band positive blocks of heterochromatin were usually about equal in size (Figure 2). In the chromosomes examined there was always G-band positive material juxtaposed around the primary constriction on both arms. The amount of this material was quite variable as were the proportions of the material from one arm to the other. Chromosomes which contained unusually large amounts of G-band positive material in the short arms exhibited an hour-glass shaped pattern when stained with C-banding and for this reason have classically been referred to as partial pericentric inversions. That these represent true inversions of material from the long arm to the short may be an invalid assumption and will be dealt with in more detail in the discussion section. Giemsa-11 heterochromatin was more distally located (usually in the long arms) than the G-band positive heterochromatin and was often surrounded by the latter. 

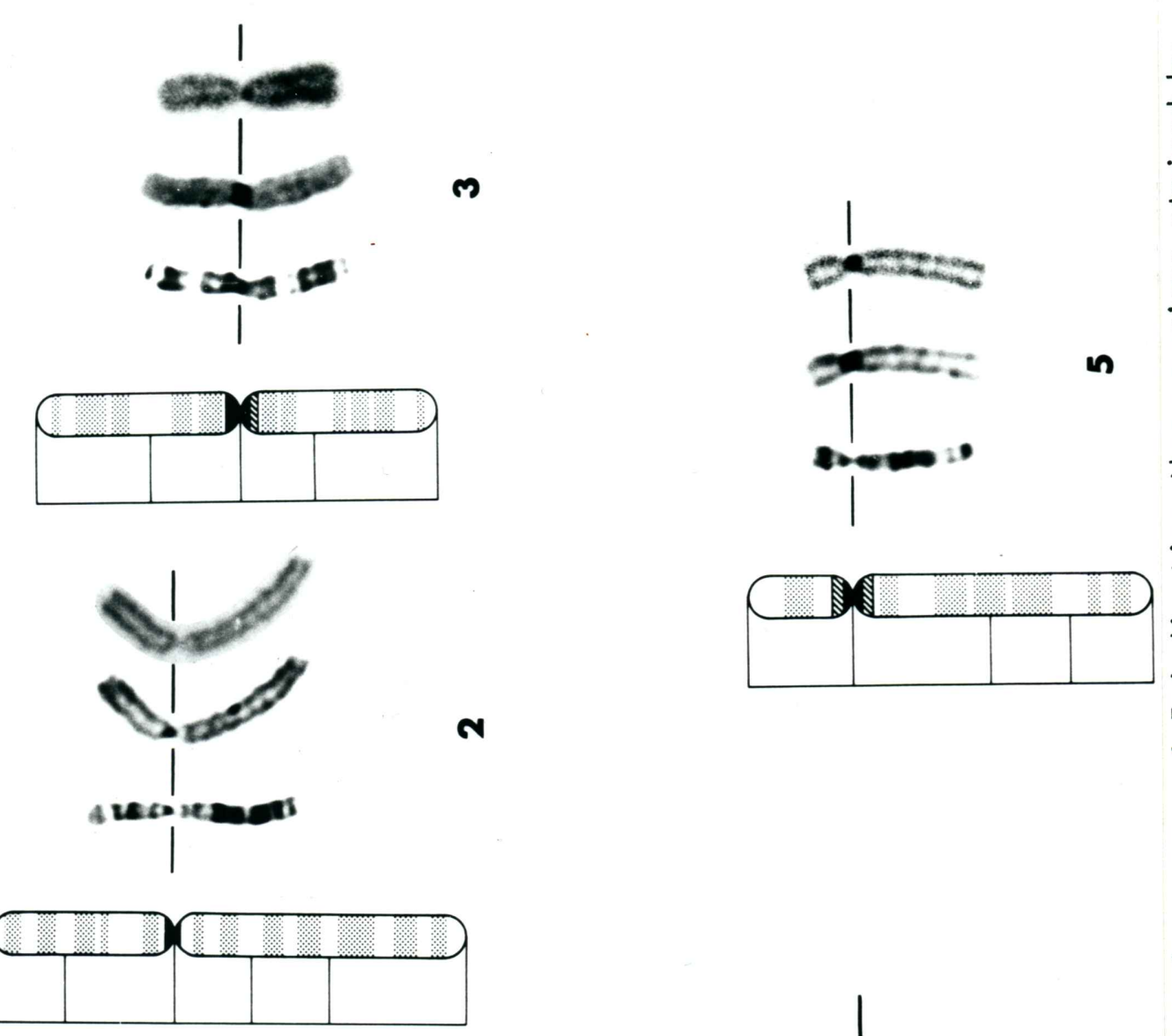

순

둔

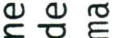

T E

फ

ธิ

등 드워

ర)

능 은

a $x+5$

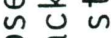

은 들 을

రण

음ㅇ

둔

๘

u

드용

$\therefore+$

드마

$1 \cdot r \quad 1$

$\rightarrow \pm 0$

थ

है등

in

능

응 어

엉 슨

ह

ه

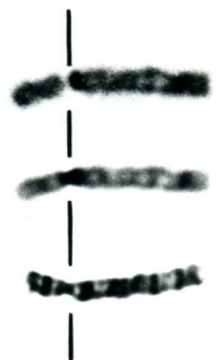

(1) 들

$\rightarrow$ थ

+थ

매

잉 응

थี ह.

E.

음 ह

$<$ 응

뜯ำ

ช ซิ

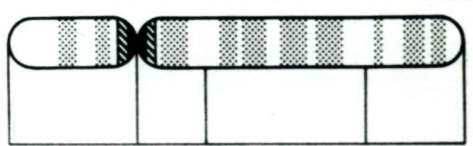

ก 1 वे

है山+

ป م ع

$0+1$ \& 4

. $4>0$ च 4 .

-

시은

교드

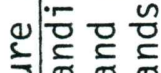

ఏํํㅇำ

تسأ 
It was not unusual to find more than one block of Giemsa-11 material on a chromosome, invariably with G-band positive material in between.

Chromosome 2. Most of the C-band region appeared to be G-band positive. Although chromosomes containing some Giemsa-11 material were encountered they were rare.

Chromosome 3. Most of the C-band region stained with G-banding. A small amount of Giemsa-11 heterochromatin was often present and more distally located on the long arm.

Chromosomes $4 \& 5$. The $\mathrm{C}$-band regions of these two chromosomes appeared to contain variable amounts of G-band and Giemsa-11 positive heterochromatin. There was as much variation between homologues as between non-homologues. Both chromosomes exhibited some G-band positive heterochromatin immediately surrounding the centromeres and often one arm contained a disproportionately larger amount. Giemsa-11 positive heterochromatin was most often located on the arm containing the least amount of G-band heterochromatin. It was not uncommon for either chromosome 4 or 5 to contain no identifiable Giemsa-11 at a11.

Chromosome 6. As depicted in Figure 3, most of the C-band heterochromatin is G-band positive. In the course of this study no Giemsa-11 material was ever found on this chromosome.

Chromosome 7. The G-band positive heterochromatin was found, mainly on the long arms and juxtacentromeric, while Giemsa-11 material was found to be localized on the short arm side of the C-band.

$\underline{X}$ Chromosome. The entire C-band region appeared to stain positive with G-banding and no Giemsa-11 heterochromatin was observed. 


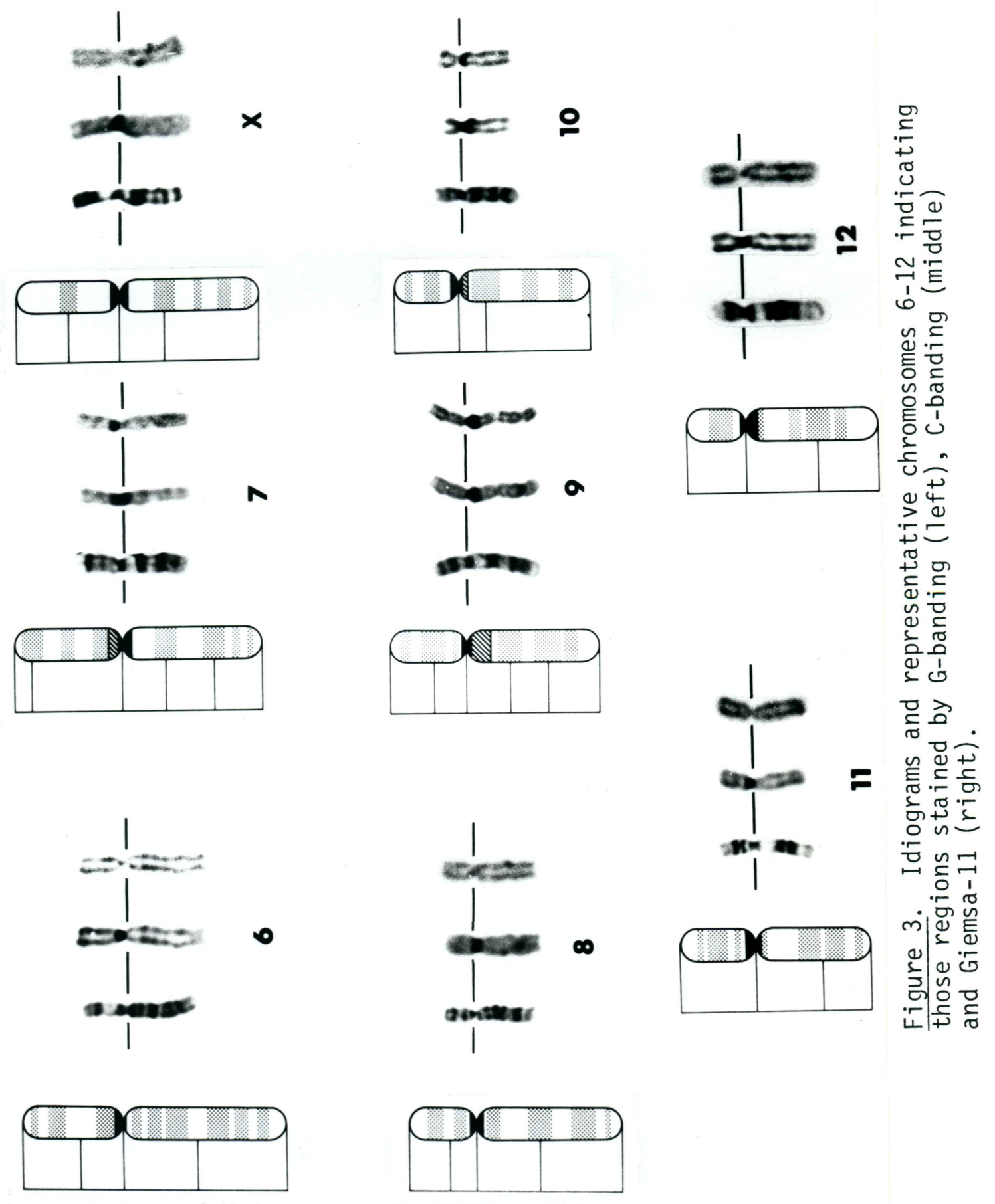


Chromosome 8. The entire C-band region stained positively with G-banding and did not stain with Giemsa-11.

Chromosome 9. This chromosome had an extremely variable heterochromatic region. The two characteristics which were consistent were that G-band positive heterochromatin immediately surrounded the centromeres and that an adjacent block of Giemsa-11 heterochromatin was more distally located. Occasionally, as in chromosome 1, several blocks of Giemsa-11 positive material were found interspersed with G-band positive heterochromatin. These extra G-bands within the C-band region have previously been reported (Madan, 1979) but not related to "segmented" appearing chromosomes identified by Giemsa-11, such as those presented here (Figure 10f).

Frequently, there were different amounts of G-band positive heterochromatin on the two sides of the centromere. Also as with chromosome 1, when the short arm of the chromosome contained a large amount of G-band positive heterochromatin the entire C-band region had an hour-glass shaped configuration; this has been referred to in the literature as having arisen by a partial pericentric inversion. This too, will be dealt with in the discussion section.

Occasionaliy chromosomes 9 were observed which contained Giemsa-11 positive material in the short arms, however, no chromosomes were observed to have this material in both arms simultaneously.

Chromosome 10. G-band positive heterochromatin was found juxtacentromeric and in the short arms, with Giemsa-11 heterochromatin present in the long arms.

Chromosome 11. G-banding denoted most of the heterochromatin, 
which was in the short arm, but some existed in the long arm as well. Chromosome 12. The C-band heterochromatin existed predominantly in the long arm and was G-band positive. No Giemsa-11 positive heterochromatin was observed.

Chromosomes $13,14,15,21 \& 22$. The heterochromatic regions of these chromosomes were very similar in their general organization while there were as many minor variations between homologues as there were in the group as a whole. G-band positive heterochromatin was seen on both sides of the centromere while Giemsa-11 positive heterochromatin was more distally located in the short arm satellite regions (Figure 4). The amounts of the two components differed markediy on different $D$-group chromosomes though a total lack of either was not found. The satellites on these chromosomes sometimes stained positive with G-banding but were most commonly G-band negative.

Chromosome 16. The entire C-band region stained positive with G-banding and contained no Giemsa-11 positive heterochromatin.

Chromosome 17. G-band positive heterochromatin was located around the centromeres with a predominance in the long arm. The Giemsa-11 heterochromatin was located in the short arm and was distal to the juxtacentromeric G-band material.

Chromosome 18. The entire C-band was G-band positive and was distributed in roughly equal amounts in the short and long arms. No Giemsa-11 staining was present.

Chromosome 19. The entire C-band region was G-band positive. The amounts located on the two arms varied greatly. Chromosomes were found containing this heterochromatin entirely on the short arm, 


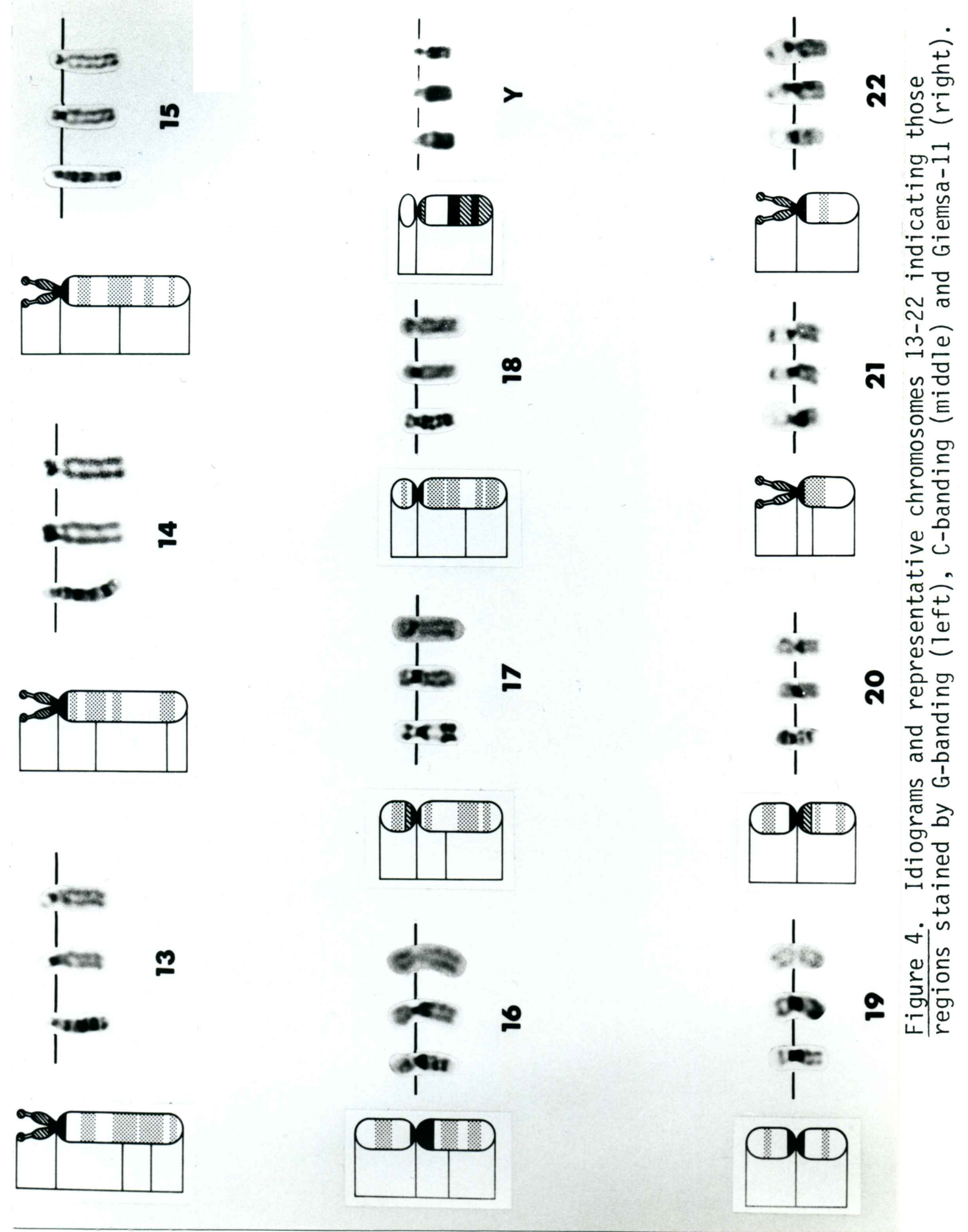


long arm or more commonly on both simultaneously.

Chromosome 20. Most of the G-band positive heterochromatin was juxtacentromeric and in the short arm while Giemsa-11 positive heterochromatin was more distal and located in the long arm.

Y Chromosome. While there was little or no G-band positive heterochromatin at the centromeres there were usually several bands located more distally in the long arm. The centromeres themselves appeared to be unstained with G-banding and there was a small amount of Giemsa-11 positive heterochromatin adjacent to the centromeres on the long arms. There was also Giemsa-11 material intermixed between the G-bands on the long arms similarly to some chromosomes 1 and 9. The $Y$ chromosome is unique in that it has little or no G-band positive heterochromatin at the centromere.

In summary, all of the chromosomes except the $Y$ contained some G-band positive heterochromatin surrouding the centromeres. The relative amounts differed and some chromosomes exhibited Giemsa-11 positive heterochromatin distal to this G-band heterochromatin. Chromosomes $1,9,16$ and the $Y$ are of special interest because they contain unusually large C-bands. Chromosome 16 exhibited exclusively G-band positive heterochromatin, chromosome 9 mostly Giemsa-11 positive heterochromatin and chromosomes 1 and the $Y$ varying amounts of both types of heterochromatin.

\section{Lateral Asymmetry}

Lateral asymmetry was found on most chromosomes but was most apparent on chromosomes $1,15,16$ and the $Y$. Figure 5 illustrates 


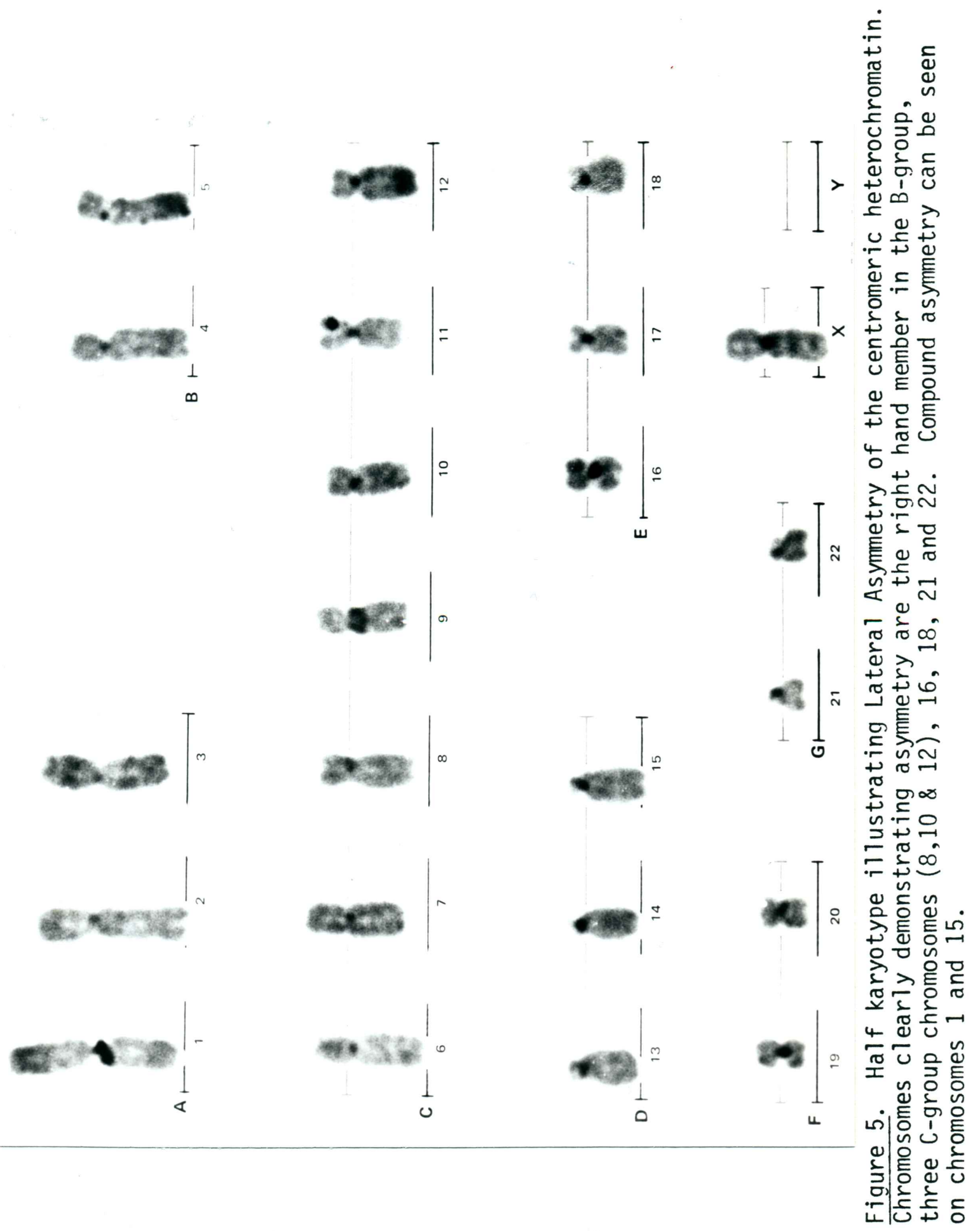


a haploid karyotype stained to reveal lateral asymmetry. The chromosome 1 reveals compound lateral asymmetry in which the dark staining heterochromatin transposes from one chromatid to the other indicating that the thymine bias in this heterchromatin has reversed or "switched" from one strand of the double helix to the other.

Chromosome 15 also appears to demonstrate compound lateral asymmetry in this case. Chromosomes which clearly demonstrate simple lateral asymmetry in this particular individual are: the right hand member in the B-group (possibly a 5), three C-group chromosomes $(8,10,12)$, 16, 18, 21 and 22. Although the $Y$ chromosome is not shown in this figure, it and chromosome 16 were found to invariably demonstrate simple lateral asymmetry. The $Y$ chromosome has two regions containing constitutive heterochromatin: one at the centromere and one on the distal portion of the long arm. While simple lateral asymetry was found on the larger distal region, no asymmetry was detected in this centromeric area, possibly because the centromeric C-band is very small.

The C-bands on chromosomes 9 appeared symmetrical when stained for lateral asymmetry, both chromatids staining darkly. Chromosomes 9 were occasionaliy observed which suggested the presence of asymmetry but the asymmetry appeared to be very complex in nature. The dark staining blocks making up the $\mathrm{C}$-band region seemed to alternate back and forth many times. Chromosomes containing an unusually large amount of G-band positive heterochromatin clearly demonstrated simple lateral asymmetry in this region but a more complex compound asymmetry (or symmetry) in the remaining Giemsa-11 positive heterochromatin (Figure 6). It is 


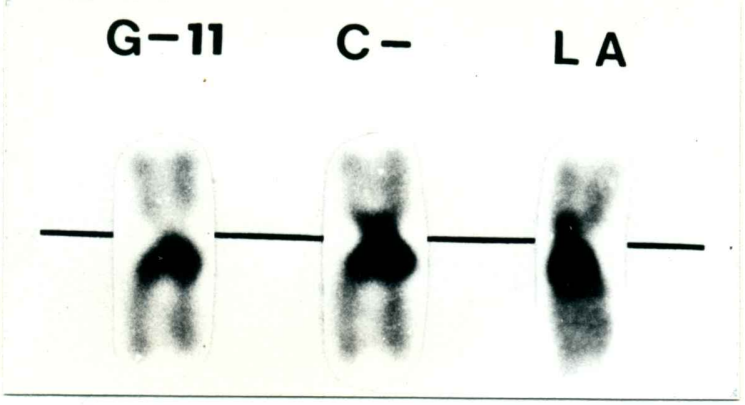

Figure 6. A chromosome 9 with an enlarged amount of Giemsa-11 negative (G-band positive) heterochromatin in the short arm. While Lateral Asymmetry exists in this short arm material it is not apparent in the Giemsa-11 material on the long arm.

\section{Lateral \\ Asymmetry \\ Giemsa-11 \\ C-Banding}

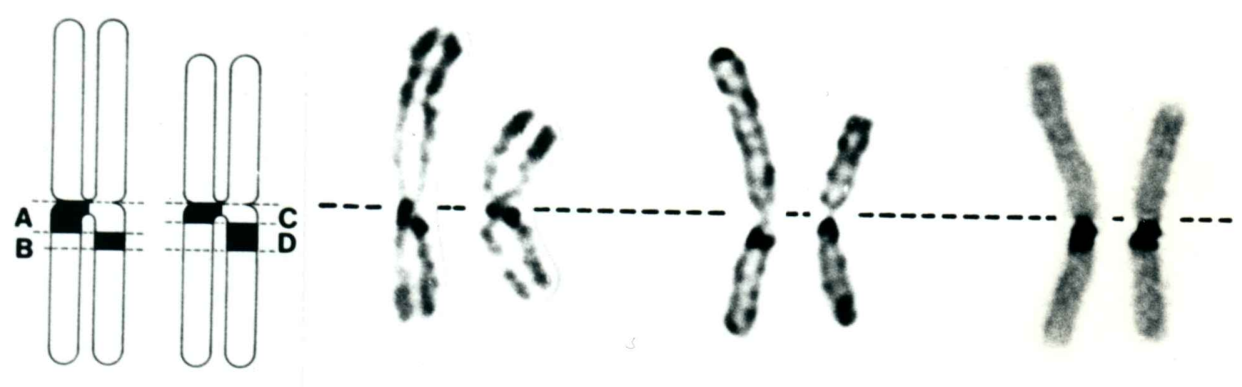

Figure 7. Homologous chromosomes 1 from an individual demonstrating a comparison between Lateral Asymmetry, Giemsa-11 and C-banding. Block "A" on the left hand chromosome correlates to the negatively staining region with Giemsa-11 while block " $B$ " correlates to the positive region. On the right hand chromosome block "C" stains with Giemsa-11 and "D" does not. The negative Giemsa-11 regions stain positive with G-banding. 
interesting that chromosome 16 revealed only simple lateral asymmetry and contained only one major type of heterochromatin (G-band positive) while chromosome 9 demonstrated simple in one region and compound lateral asymmetry in another and contains two major types of heterochromatin. This suggests that Giemsa-11 heterochromatin may have undergone more changes in T-bias than G-band positive heterochromatin. Chromosome generally contained equal quantities of both G-band positive and Giemsá-11 positive heterochromatin and usually demonstrated a compound lateral asymmetry involving two blocks of alternating thymine bias. Upon comparing C-banded, Giemsa-11 and lateral asymmetry stained preparations of the same chromosomes 1 it became apparent that the Giemsa-11 positive heterochromatin corresponded in size and location to one of the blockș stained by the lateral asymmetry technique while the Giemsa-11 negative heterochromatin (which would be G-band positive) corresponded to the other block (Figure 7). One of the chromosomes in this figure has a portion of euchromatin from a 15 translocated onto it and was used to unequivocally differentiate the two homologues as the staining techniques were not sequentially performed on the same preparation. The relationship presented above strongly suggests that on chromosome 1 and possibly others the two types of heterochromatin contain opposite thymine biases. This is in contrast to the $Y$ chromosome in which several types of heterochromatin may be intermixed and yet it invariably reveals simple lateral asymmetry.

Chromosomes 1 have also been observed to contain three and sometimes four blocks of differing thymine bias when stained to reveal lateral asymmetry. In all cases examined G-band positive heterochromatin 


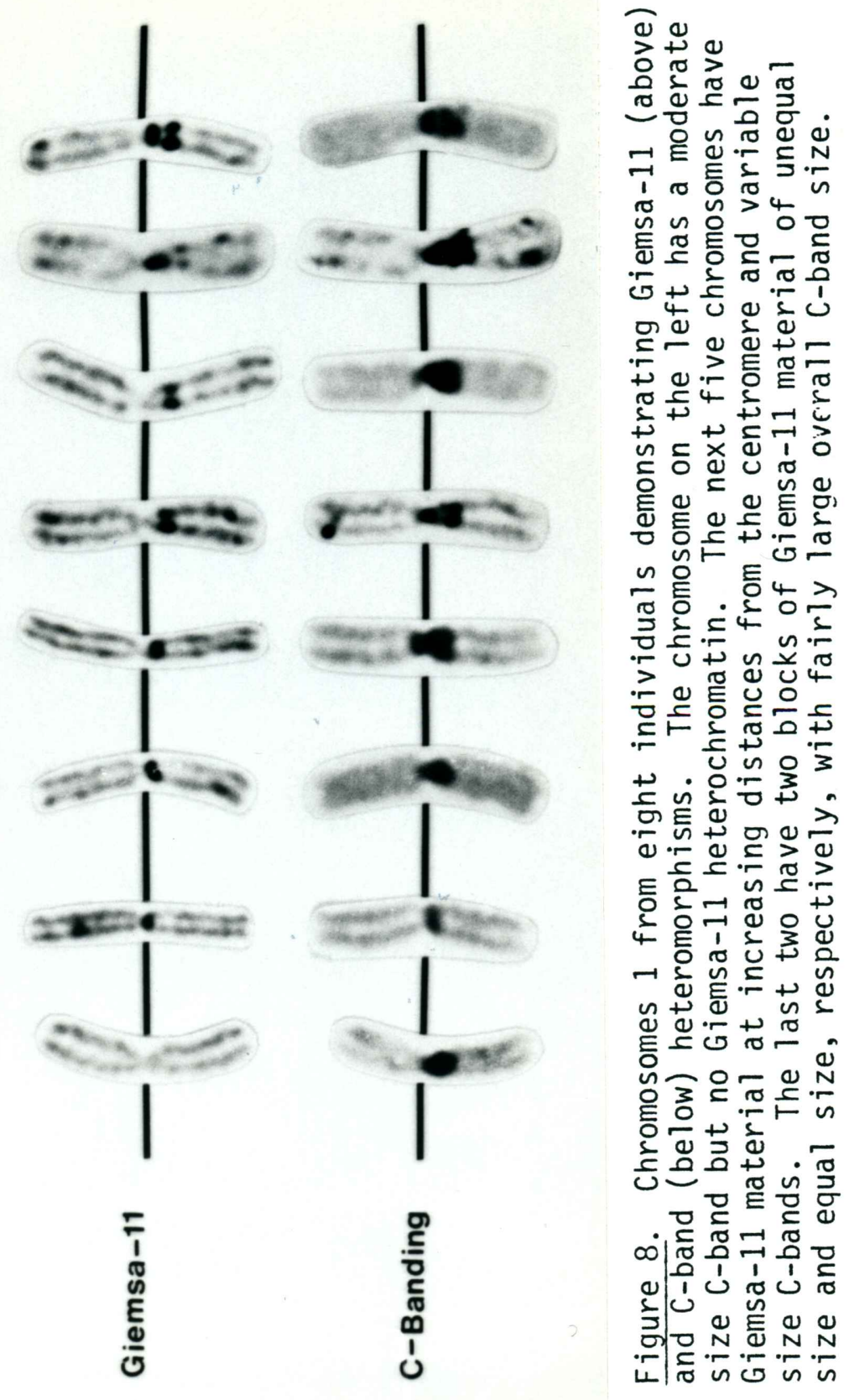


correlated to the other blocks. This finding strongly suggests that compound lateral asymmetry may be more frequent in the latter type of heterochromatin. This would help to explain why chromosome 9, which generally contained quite large amounts of Giemsa-11 positive heterochromatin consistently revealed lateral symetry, being so complex as to stain both chromatids darkly.

\section{Variations in C-band Size and Organization}

The Cd, Giemsa-11 and G-banding techniques permit one to differentiate subtypes of constitutive heterochromatin within the $\mathrm{C}$-band regions of many chromosomes. These tools offer valuable assistance in ascertaining chromosome rearrangements and examining the production of $\mathrm{C}$-band heteromorphisms. Special attention was paid to those chromosomes containing large amounts of constitutive heterochromatin and both Giemsa-11 and Gband positive material, notably chromosomes 1, 9 and the $Y$. Chromosome 16 was not especially informative in this respect because though it generally contains a fairly large C-band region, it stains with only $\mathrm{Cd}$ and G-banding. The Cd technique alone revealed only a limited amount of heteromorphism on most chromosomes. Occasionally there was an increase in amount on chromosomes 6 and a suggestion of two or more sets of dots on enlarged chromosomes 1,9 and 16 .

Giemsa-11 and C-banding were performed sequentially on the same preparations and the chromosomes 1 compared. Figure 8 is a composite of eight chromosomes 1 , differing in sizes of the C-bands. The first chromosome on the left contains a fairly average size C-band but no 


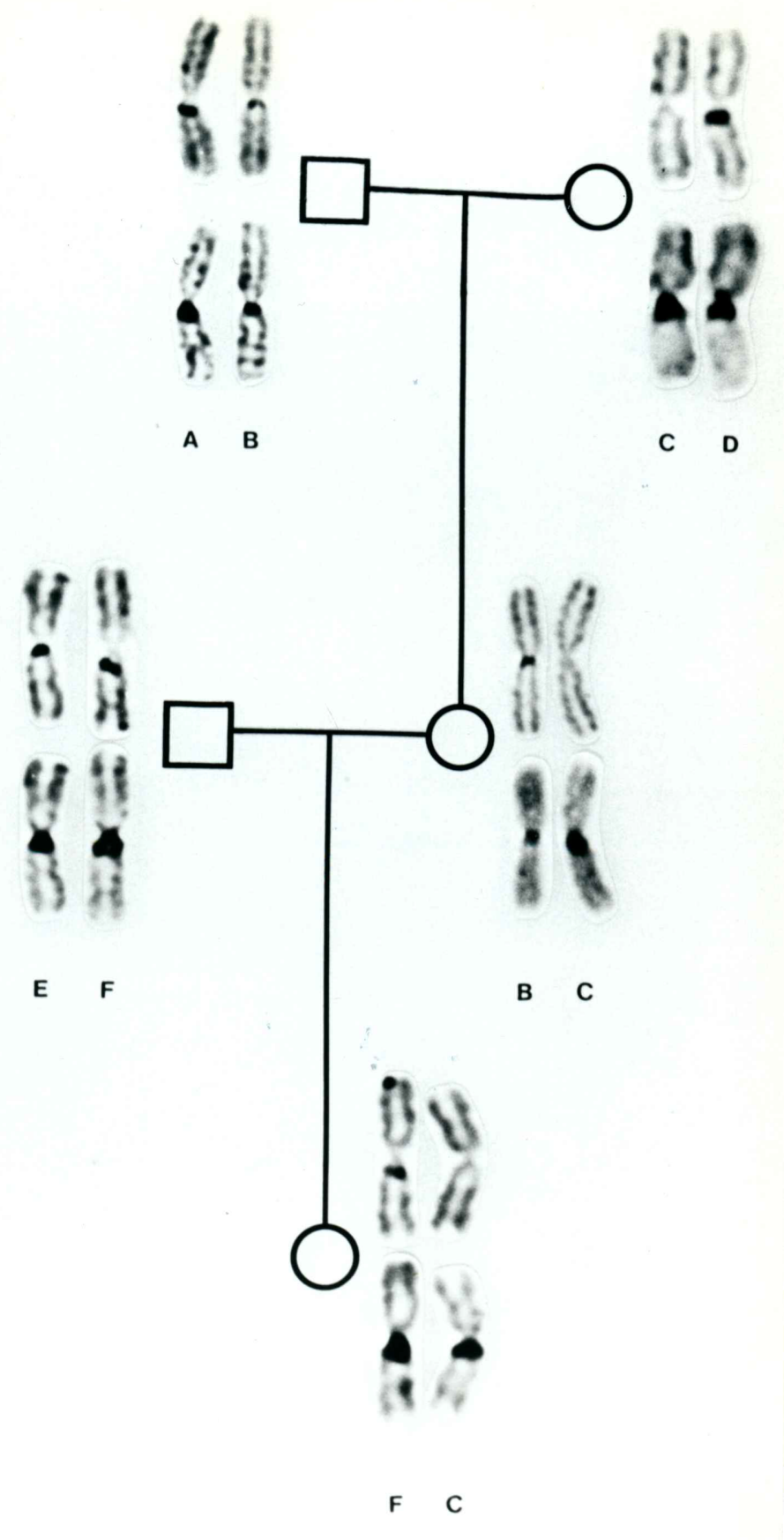

Figure 9. Heritability of Giemsa-11 and C-banded chromosomes 1. The Giemsa-11 stained chromosomes appear as the upper pair while the sequentially $C$-banded chromosomes appear below and are labeled "A, B, $C, D, E$ and $F$ " for reference. Note that chromosome " $C$ " appears in three generations and contains no noticable Giemsa-11 heterochromatin. Other chromosomes such as "E" and "F" are not readily discernible with C-banding but may be distinguished by the proximity of the Giemsa-11. 
Giemsa-11 positive heterochromatin whatsoever. The next five chromosomes have blocks of Giemsa-11 heterochromatin at increasing distances from the centromeres and variable overall sizes of C-band heterochromatin. The last two chromosomes 1 to the right have enlarged $\mathrm{C}$-bands and two blocks of Giemsa-11 material; one with two blocks of equal size, the other with two blocks of unequal size. On the chromosomes with enlarged C-bands the overall organization of the subtypes was not the same as that of the average sized C-bands but appeared to be duplications of a smaller simpler pattern. This is to say that rather than one block of Giemsa-11 material being enlarged several smaller blocks were present. Other chromosomes 1 with enlarged C-bands, although not shown here, were found to contain only one block of average size Giemsa-11 material. Most of the heterochromatin not stained by the Giemsa-11 technique was found to be G-band positive. Other examples are presented in Figure 11.

Figure 9 demonstrates that Giemsa-11 variants on chromosome 1 are inherited in simple codominant Mendelian fashion (labeled A, B, $C, D, E$ and $F$ for reference). Chromosome " $C$ " is the chromosome in Figure 9 which contained no Giemsa-11 material and was transmitted for at least three generations. The Giemsa-11 stained chromosomes are the upper pairs and were sequentially $\mathrm{C}$-banded for direct comparisons, which are the lower pairs of chromosomes.

Chromosomes 9 were examined with G-banding, Giemsa-11 and C-banding and were also informative in elucidating differences in C-band variability. As with chromosome 1, chromosome 9 generally contained G-band positive heterochromatin surrounding the centromeres and a more distally located 


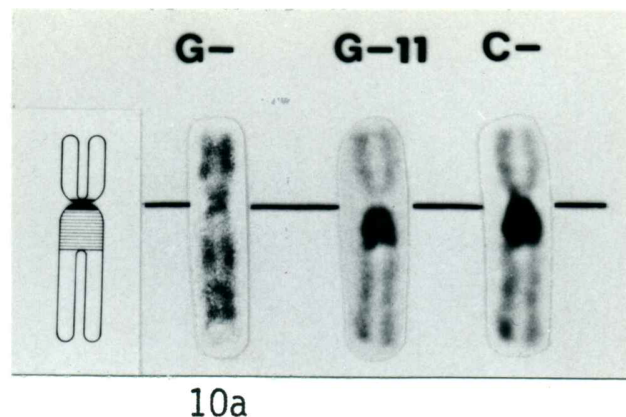

$10 \mathrm{a}$

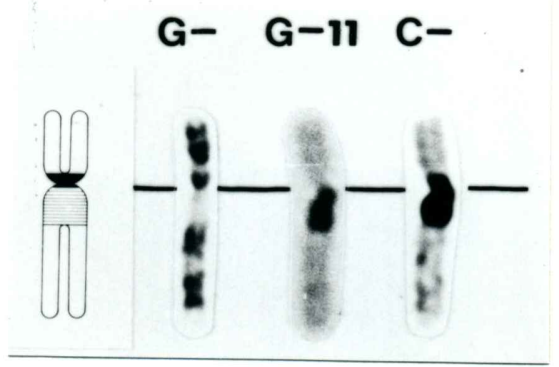

$10 \mathrm{c}$

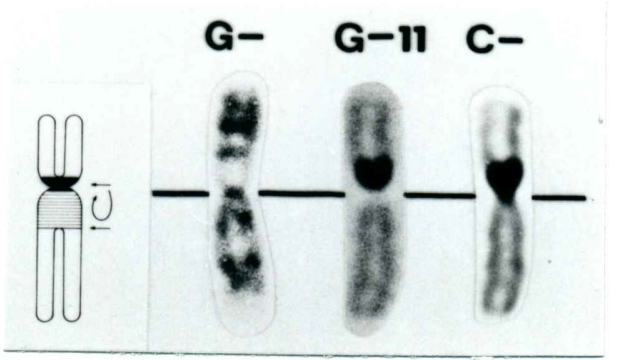

$10 \mathrm{e}$
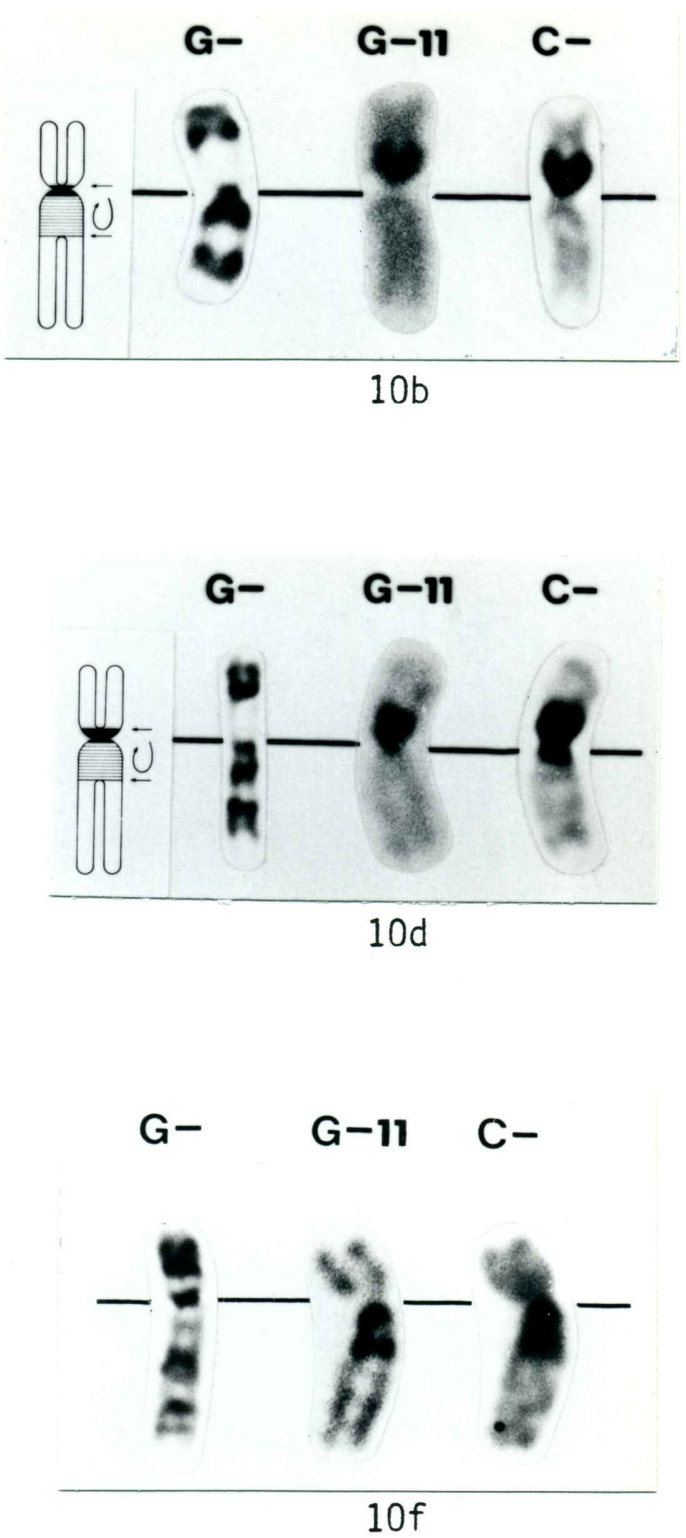

Figure 10a-f. (a) An average chromosome 9 stained with G-banding, Giemsa-11 and C-banding. Idiogram to the left depicts G-band (solid black) and Giemsa-11 (cross-hatched) heterochromatin. (b) A chromosome 9 which has apparently undergone a pericentric inversion of the C-band heterochromatin. (c) A chromosome 9 with an enlarged amount of G-band heterochromatin on the short arm. (d) A chromosome which could have arisen from one similar to that in (c) via a pericentric inversion. (e) A chromosome similar to that in (d) but with two blocks of G-band positive heterochromatin. (f) A chromosome 9 with a segmented appearance with Giemsa-11 and an extra G-band within the heterochromatic region which corresponds to the achromatic segment. See text. 
block or blocks of Giemsa-11 positive heterochromatin. In this case the Giemsa-11 material was usually much larger than that of chromosome 1. Figure 10a compares the G-, C-banding and Giemsa-11 patterns of a typical chromosome 9. The idiogram to the left illustrates the G-band positive (solid black) and the Giemsa-11 positive (cross-hatched) heterochromatin. Figure 10b demonstrates a chromosome 9 with the majority of its C-band heterochromatin on the short arm. The intra C-band organization is preserved and similar to that in Figure 10a: the G-band material is at the centromeres and the Giemsa-11 material is more distally located. The mechanism by which this chromosome may have formed will be presented in the discussion section. Figure 10c illustrates a chromosome with C-band heterochromatin in both the short and long arms. This chromosome would be similar to any other 9 except that the G-band positive heterochromatin on the short arm side of the centromere is greatiy enlarged. Figure 10d illustrates a chromosome that also has $\mathrm{C}$-band heterochromatin on both arms except the Giemsa-11 material is on the short arm and an enlarged amount of G-band positive heterochromatin is on the long arm. Figure 10e shows a chromosome which contains most of its C-band heterochromatin on the short arm. Interestingly in this chromosome there are two distinct blocks of G-band positive heterochromatin, one surrounding the centromeres and the other located in the short arm on the distar side of the Giemsa-11 heterochromatin.

Chromosomes 9 often exhibit a segmented appearance with Giemsa-11 staining. A pale or unstained band separates blocks of heavily staining Giemsa-11 positive material. Madan (1978) has reported chromosomes 9 which contain extra dark bands when stained for G-banding. Comparisons 


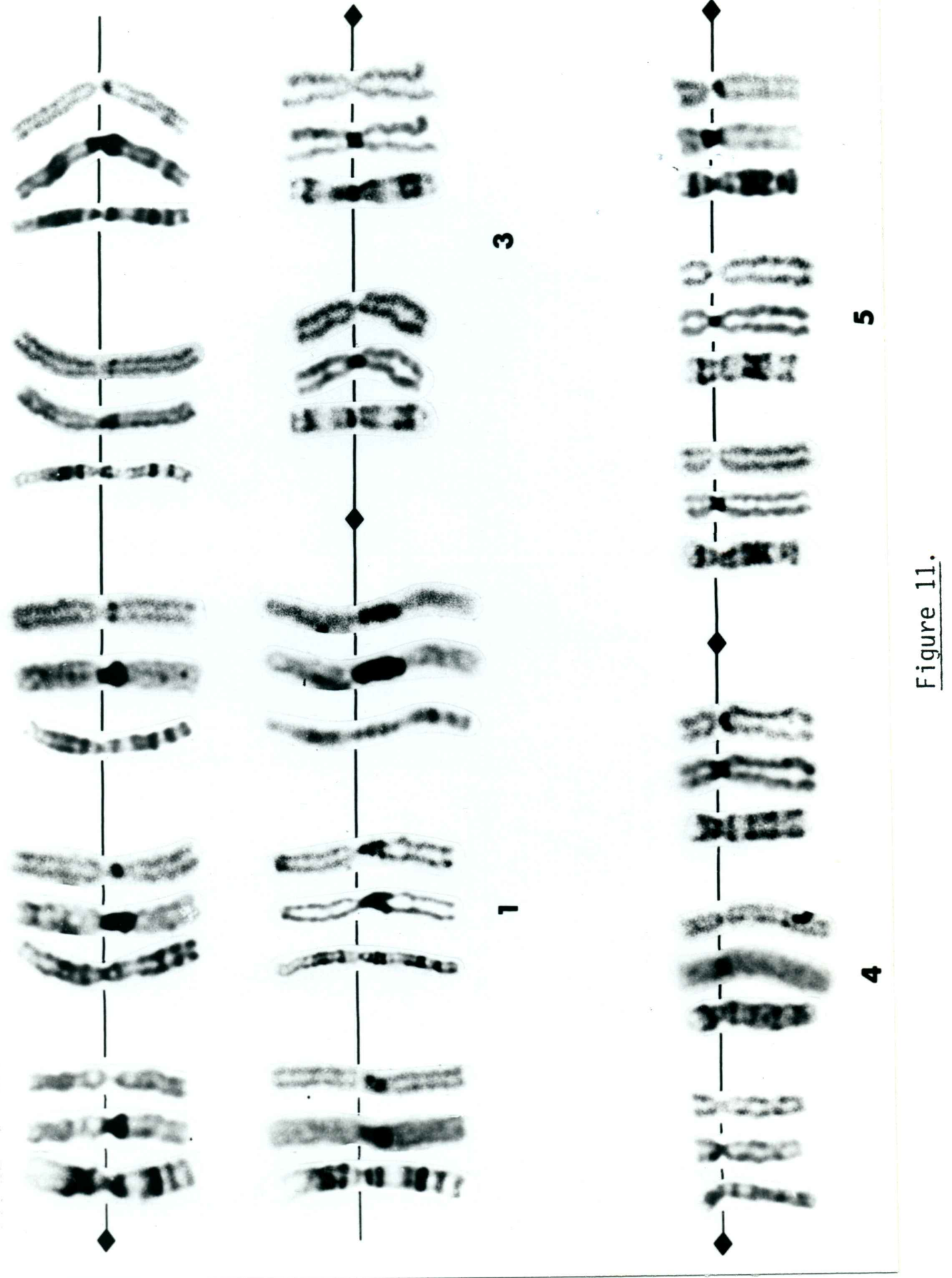


of such chromosomes with the two techniques reveals that this extra G-band corresponds in size and location to the achromatic band observed with Giemsa-11 staining. This extra G-band which stained positive with $\mathrm{C}$-banding indicates it has properties similar to that of the G-band positive heterochromatin surrounding the centromeres.

A11 of the chromosome 9 variants presented above were also observed in many other unrelated individuals in this study and therefore are not considered to be rare chromosome variants.

Y chromosomes, which are not illustrated, were found to contain alternating blocks of G-band and Giemsa-11 positive heterochromatin in the distally located heterochromatin. Generally there were two blocks of each and the two blocks of similarly stained material usually appeared to be of equal sizes.

Other Chromosome Variants

Figures 11, 12 and 13 illustrate other examples of chromosome variants encountered during the course of this study. The G-banded chromosomes are to the left, the C-banded in the center and the Giemsa-11 to the right of each triad. Of the eight chromosomes 1 in Figure 11 the first one contains a C-band with no Giemsa-11 heterochromatin and is entirely stained by G-banding; the next three have Giemsa-11 material at varying distances from the centromeres; the fifth 1 has an enlarged amount of G-band heterochromatin on the short arm; the next has two blocks of Giemsa-11 heterochromatin and the last two have three blocks each of Giemsa-11 heterochromatin. The Giemsa-11 staining corresponds to the light regions produced between the blocks 


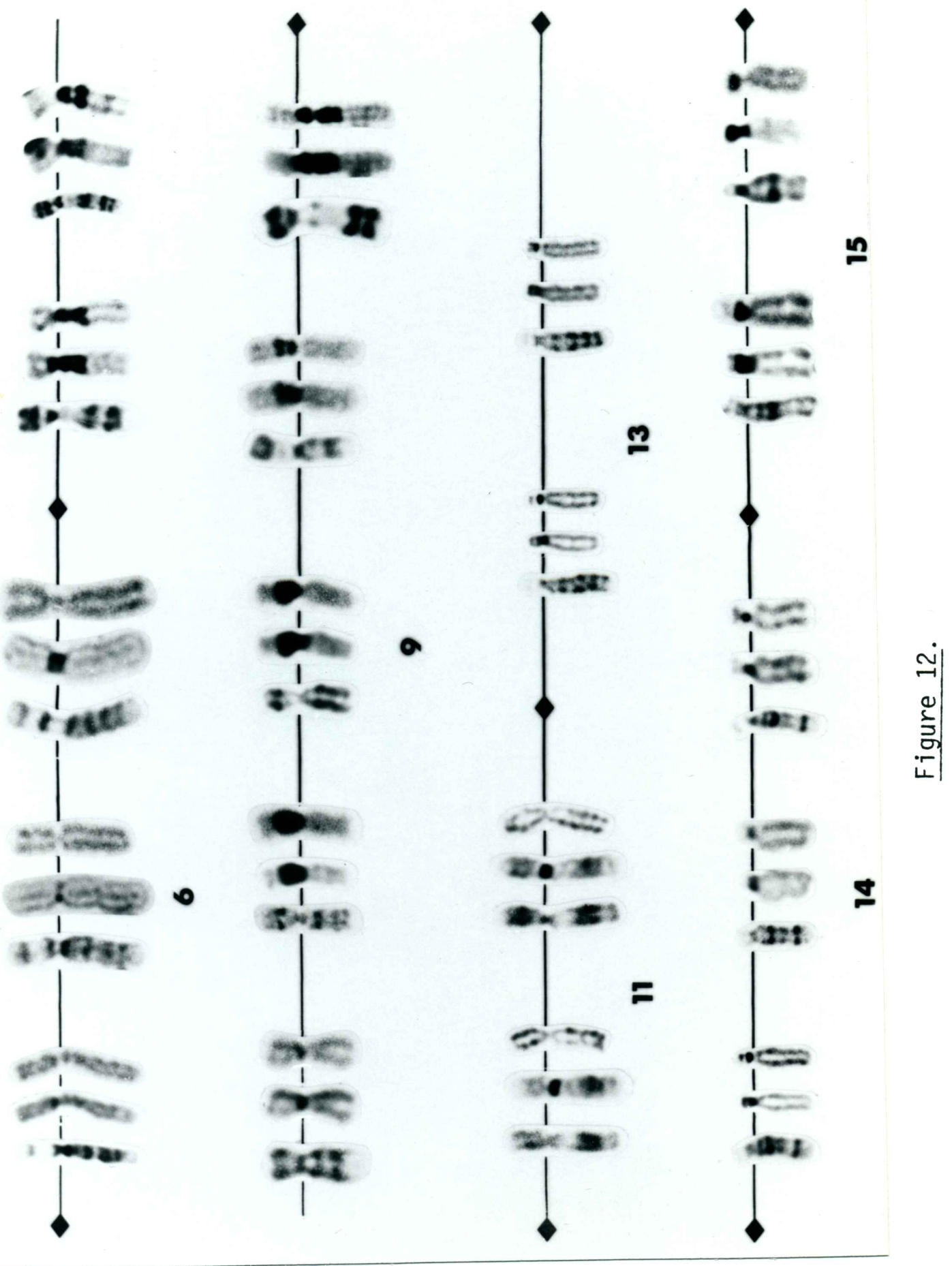


of G-band positive heterochromatin. Two chromosomes 3 are illustrated in Figure 11. The 3 on the left has a small amount of Giemsa-11 material on the long arm while the 3 on the right has none. Also in the figure are three each of chromosomes 4 and 5 containing no Giemsa-11 positive heterochromatin (left), some in the short arm (middle), or long arm (right) respectively.

Three chromosomes 6 with increasing C-band sizes are shown in Figure 12. Although these chromosomes do not contain Giemsa-11 positive heterochromatin they do contain quantities of another type of G-band negative heterochromatin, shown to be $\mathrm{Cd}$ positive in Figure 1 as discussed earlier. Seven chromosomes 9 with unusual staining heterogeneities are also shown. The first 9 contains an enlarged amount of G-band positive heterochromatin on the short arm; the next, segmented Giemsa-11 staining regions interspersed by G-band positive heterochromatin; the third has a much reduced amount of Giemsa-11 material on the short arm; the fourth and fifth chromosomes have what appear to be pericentric inversions but with differing amounts of G-band heterochromatin; the sixth 9 is not only inverted but reveals segmentation with Giemsa-11 staining and the seventh has a similar segmentation but of different sized blocks of Giemsa-11. Next, in Figure 12 are two chromosomes 11; the first has $\mathrm{C}$-band material on the long arm and the second has it on both long and short arms. Also in the figure are chromosomes 13, 14 and 15 with different amounts of the two types of heterochromatin. Note that there is always some G-band positive heterochromatin surrounding the centromere and that Giemsa-11 heterochromatin is more distally located on the short arms. 


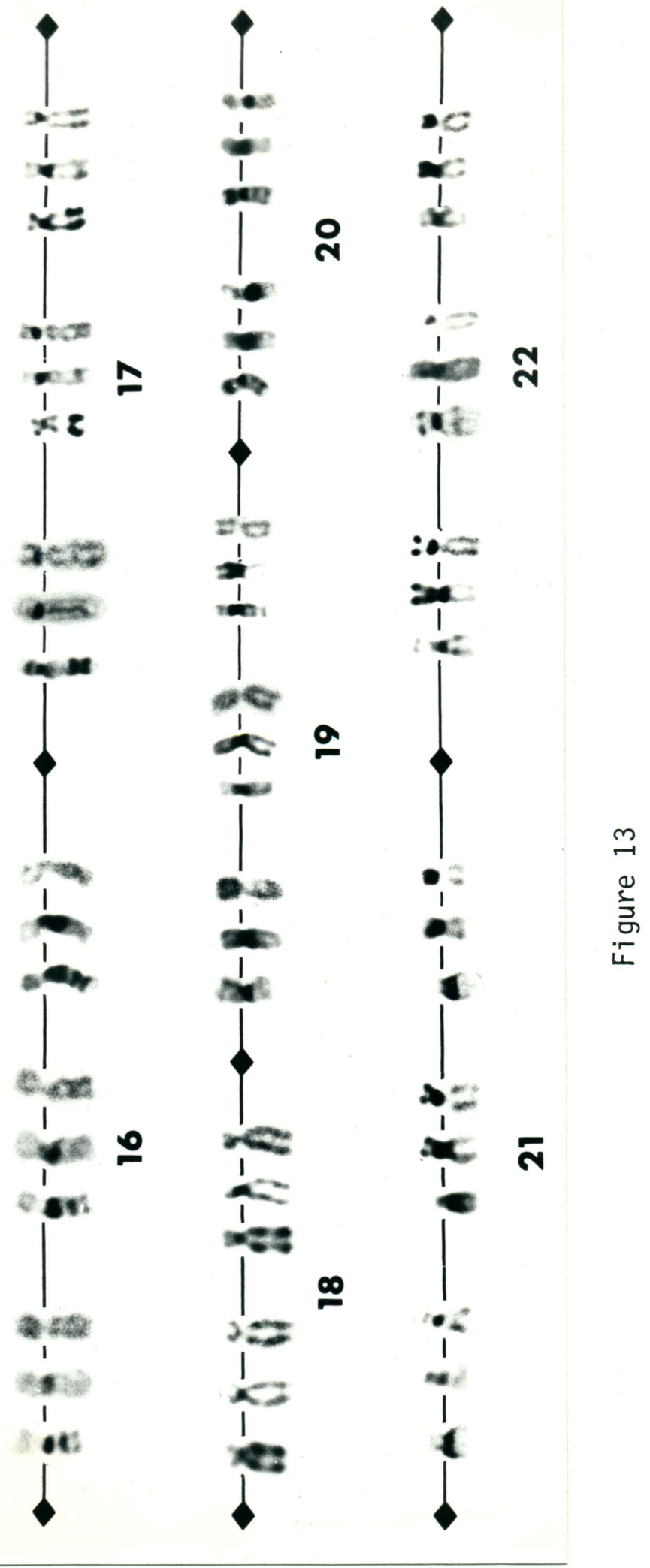


Figures 11, 12 and 13. Examples of heteromorphic chromosomes 1, 3, $4,5,6,9,11,13,14,15,16,17,18,19,20,21$ and 22 . The G-banded chromosomes are located on the left of each triplet, the C-banded in the middle and the Giemsa-11 stained to the right.

In Figure 11 are eight chromosomes 1. The first (upper left hand) contains no Giemsa-11 heterochromatin but stains with G-banding; the next three have blocks of Giemsa-11 at increasing distances from the centromere. The fifth chromosome has an enlarged amount of G-band heterochromatin in the short arm with eparts it with an hour-glass shape with C-banding. The next three chromsomes (second row) have two, three and three blocks of Giemsa-11 respectively. The Giemsa-11 positive blocks correlate to the G-band negative areas. Next are presented chromosomes 3 with a small amount of Giemsa-11 (left) and no Giemsa-11 staining ( $r$ ight). The left hand 4 has no Giemsa-11, the second has some in the short arm and the third has Giemsa-11 material in the long arm. The same is true of the chromosomes 5.

Figure 12. Three chromosomes 6 with increasing amounts of G-band negative heterochromatin which does not stain with Giemsa-11 (Cd positive). Next are seven chromosomes 9. The first has an enlarged amount of G-band heterochromatin in the short arm and an hour-glass shape with C-banding. The second has a segmented appearance with Giemsa-11 which was discussed. The third has almost no detectable Giemsa-11 material. The next three have inversions of the C-bands with one revealing a segmented Giemsa-11 block(s) and the last is a chromosome 9 which is greatly enlarged and has at least two blocks of Giemsa-11 material which appear segmented. The chromosome 11 on the left has most of its C-band heterochromatin in the long arm while the one on the right has it mainly on the short. The next are chromosomes 13, 14 and 15 with increasing sizes of short arms.

Figure 13. Three chromosomes 16 with increasing sizes of C-bands, three chromosomes 17 with increasing sizes of G-band heterochromatin, two 18s with increasing sizes of C-bands (G-band positive), three chromosomes 19 with G-band heterochromatin in long arms, both the arms and the short arm respectively, chromosomes 21 and 22 with increasing lengths of short arms. 
Three chromosomes 16 of increasing C-band size are shown in Figure 13, along with three chromosomes 17 , with increasing amounts of G-band positive heterochromatin on the long arms; two chromosomes 18, with increasing C-band size; three chromosomes 19, with G-band positive heterochromatin in the long, long, and short and short arms respectively; chromosomes 20, with decreasing amounts of Giemsa-11 positive heterochromatin; chromosomes 21 and 22 with differing amounts of the two components and similar in organization to chromosomes 13,14 and 15.

Some general features which become evident are that G-band and Giemsa-11 heterochromatin may vary in size and location and that they seem to do so independently of one another, i.e., an increase in the amount of one does not necessarily predispose to an increase in the other. There is a very strong suggestion that G-band heterochromatin exhibits much more variation in amount than Giemsa-11 heterochromatin. Though size discrepancies certainly exist for Giemsa-11 heterochromatin, those chromosomes which contain larger amounts of it usually exhibit it as several discrete blocks, each of average size as though the changes involve quantum increases of this material. In this respect it would seem likely that the variation in amount of the two different components is brought about in two different manners. 


\section{DISCUSSION}

This study demonstrated that G-band positive heterochromatin invariably revealed simple lateral asymmetry while Giemsa-11 positive heterochromatin exhibited both simple and compound lateral asymmetry and that on chromosome 1, at least, there was usually a change in thymine bias between juxtaposed G-band and Giemsa-11 positive heterochromatin. By comparing the lateral asymmetry results presented here with previously published information on satellite DNA localizations it can be postulated that regions of heterochromatin which correlate with satellite II reveal simple lateral asymmetry while regions containing several satellite DNAs reveal compound lateral asymmetry. By examination of Table II one can see, for example, that chromosome 16 contains exclusively satellite II and invariably demonstrates simple lateral asymmetry while chromosome 9 contains large amounts of all four satellites and reveals a complex compound lateral asymmetry. At this point it cannot be determined whether compound lateral asymmetry on all chromosomes is a direct result of having several different satellite DNAs of opposite thymine bias or whether this reflects changes in thymine bias within a given type of DNA. On chromosome 1, at least, it would seem that compound lateral asymmetry can exist within Giemsa-11 positive heterochromatin and Giemsa-11 staining possibly correlates with the presence of satellite II DNA (Bühler, et. a. , 1975). 


\section{TABLE II}

COMPARISONS OF CHROMOSOMAL STAINING

AND SATELLITE DNA LOCALIZATIONS

\begin{tabular}{|c|c|c|c|c|c|c|c|}
\hline \multirow[t]{2}{*}{ Chromosome } & \multicolumn{4}{|c|}{ Satellite DNA* } & \multirow{2}{*}{$\begin{array}{c}\text { Giemsa-11 } \\
\text { Heterochromatin }\end{array}$} & \multirow[t]{2}{*}{$\underline{G-B a n d}$} & \multirow{2}{*}{$\begin{array}{l}\text { Lateral } \\
\text { Asymmetry }\end{array}$} \\
\hline & $\bar{I}$ & II & III & IV & & & \\
\hline 1 & + & $+t$ & $+t$ & t+ & ++ & t+ & Usually compound \\
\hline 2 & & & & & \pm & + & Simple \\
\hline 3 & & & & & \pm & + & Simple \\
\hline 4 & & & & & ++ & ++ & Simple or complex \\
\hline 5 & + & & + & + & $+t$ & t+ & Simple or complex \\
\hline 6 & & & & & - & + & \\
\hline 7 & + & + & + & + & + & + & \\
\hline 8 & & & & & - & + & Simple \\
\hline 9 & ++ & ++ & ++ & $+t$ & t+t & ++ & Complex compound \\
\hline 10 & & & + & + & + & + & Simple \\
\hline 11 & & & & & - & + & Simple \\
\hline 12 & + & & & & - & + & Simple \\
\hline 13 & $+t$ & & $+t$ & t+ & $+t$ & + & Compound \\
\hline 14 & ++ & + & $+t$ & t+ & ++ & + & Compound \\
\hline 15 & ++ & $+t$ & ++ & $+t$ & ++ & + & Compound \\
\hline 16 & & ++ & & & - & ++ & Simple \\
\hline 17 & & ++ & + & $+t$ & + & + & Compound \\
\hline 18 & & & & & - & + & Simple \\
\hline 19 & & & & + & - & ++ & \\
\hline 20 & + & + & ++ & $+t$ & + & + & \\
\hline 21 & ++ & ++ & + & ++ & ++ & + & Simple \\
\hline 22 & $+t$ & $+t$ & ++ & $+t$ & ++ & + & Simple \\
\hline$x$ & & + & & & - & + & \\
\hline$Y$ & ++ & ++ & ++ & ++ & +++ & +++ & Simple \\
\hline
\end{tabular}

* (Gosden et. a1., .1975) 
For a given type of DNA, with a consistent thymine bias, to go from having simple to compound lateral asymmetry would require that a portion of the DNA undergo a $180^{\circ}$ inversion which would essentially reverse the thymine bias in the inverted segment. Kurnit (1979) believes that heterochromatin is amplified through unequal mitotic crossing over during DNA synthesis. He suggests that the majority of amplificational events conserve the thymine bias but that a rarer inversional event in which thymine bias is reversed is also possible. If one assumes that this rare event is equaliy probable for different types of DNA then one might suspect that there would be a higher incidence of compound lateral asymmetry in the "older" DNAs. Jones (1977) has suggested that a linear relationship exists between sequence divergence within a given repeated DNA and the "age" of that DNA. The "age" refers to the time elapsed since the initial amplification of that DNA. Based on this relationship he gives ages of three of the satellite DNAs as: 18-24 million years for satellite I, 6-8 million years for satellite II and 30-40 million years for satellite III. These data correlate closely with the findings that chromosome 16 contains exclusively G-band positive heterochromatin, satellite II (the youngest) and invariably reveals simple lateral asymmetry while chromosome 9 contains mostly Giemsa-11 positive heterochromatin, large amounts of all four satellites (several old and one young) and reveals mostly, what is interpreted, compound lateral asymmetry with simple lateral asymmetry in the G-band material.

Comparisons of lateral asymetry and other banding patterns on chromosomes 1 which contained increased amounts of heterochromatin on the short arms also furnished information relating to chromosome structure. 
These chromosomes have classically been referred to as "partial pericentric inversions" but this study suggests that this explanation may be invalid for the following reasons: this heterochromatin extending from the main portion of heterochromatin into the short arm was invariably G-band positive and never contained Giemsa-11 positive material. If these are truly random inversions then one might expect to find at least occasionally some inversions encompassing both types of heterochromatin. The lateral asymmetry on these chromosomes was found to be simple throughout this G-band positive heterochromatin across the centromere. It is therefore highly unlikely that these regions have arisen by a partial pericentric inversion. This argument is true also for chromosome 9. Chromosomes which contained Giemsa-11 heterochromatin in the short arms appeared to have arisen by total pericentric inversions of the C-band regions of chromosomes with enlarged amounts of G-band positive heterochromatin and not by partial pericentric inversions. Those with Giemsa-11 material in the long arm but increased amounts of G-band heterochromatin are probably not inversions at al1. Chromosomes were not found containing Giemsa-11 positive heterochromatin on both arms. For these reasons it is believed that of the chromosomes 9 presented in Figure 10, b, d and e represent total pericentric inversions of the $\mathrm{C}$-band regions while a, $c$ and $f$ designate chromosomes more commonly encountered which do not appear to be inversions. Breakpoints on most of the inverted chromosomes encountered in this study generally occurred at the heterochromatin/euchromatin junctions but occasionally some were encountered which involved breakpoints within G-band positive heterochromatin such as the case in Figure 10e. 
Apparent duplications of the $\mathrm{C}$-band region were also encountered which contained several blocks of Giemsa-11 positive heterochromatin with G-band positive heterochromatin interposed (Figure 10f). This extra G-band is thought to arise from the centromeric G-band which would indicate that here too the breakpoint was within the G-band positive heterochromatin.

\section{Possible Mechanisms of Chromosome Variation}

Observations made in this study indicate that the different types of constitutive heterochromatin can vary in two different ways. One involves a gradual change in the amount of a given type (sequence?) of DNA such as the G-band heterochromatin found near the centromeres on most chromosomes. This would be a process by which the interstrand thymine bias is conserved and would result in simple lateral asymmetry. The other involves a sudden duplication or quantum increase of several types of heterochromatin in regions such as those found often on chromosomes 1,9 and the distal two-thirds of the $Y$. This latter consequence results in the addition or duplication of several different types of DNA simultaneously (G-band and Giemsa-11) and often adds to the complexity of the region revealed by lateral asymmetry.

Meiotic Origin. It has been suggested by Craig-Holmes et. al. (1973) that unequal crossing over during meiosis plays an important role in the production of large or duplicated C-band regions. This presumabiy is accomplished by misalignment of the homologous chromatids during pairing and subsequent crossing over to produce duplicated/deficient amounts of heterochromatin between the two chromatids. Unequal cross 
overs encompassing large portions of the C-band might result in duplication of both the G-band and Giemsa-11 heterochromatin. These duplication events would produce two similar segments of heterochromatic organization when involving homologous chromosomes of similar C-band organization and dissimilar segments when homologue organizations are different.

Unequal meiotic crossing over in heterochromatin may be assumed to be extremely rare because careful cytology demonstrates early repulsion of chromosomes and a lack of chiasmata in heterochromatic segments suggesting that they seldom pair (John, 1976). Hungerford et. al. (1972) have examined human meiotic preparations using electron microscopy and observed a C-group chromosome (later found to be chromosome 9; Page, 1973) which displayed some interesting features. The homologues appear to be paired for the most part but exhibited regions around the centromeres which were not paired. In the unpaired regions there were two loose networks of chromatin, one per chromosome, looping away from each other and containing denser bodies within which have been termed "parameres". These paramere-containing networks have been shown to be Giemsa-11 positive by Page and it is probably safe to assume, based on data in this study, that the remainder of the $\mathrm{C}$-band is G-band positive. By examination of these photographs it is apparent that the centromeric or G-band positive heterochromatin is paired, while the Giemsa-11 portions are not, suggesting that the two types of heterochromatin may behave quite differently during meiosis. If unequal crossing over does occur during meiosis one might expect misalignment and breakage to occur more frequently in regions which pair such as this G-band positive heterochromatin, while the unpaired 
Giemsa-11 heterochromatin, being interposed between blocks of G-band heterochromatin, might be transposed to the homologous chromosome as a discrete unit.

Mitotic Origin. Mitomycin C, a bifunctional alkylating agent which crosslinks DNA, is known to dramatically increase the frequency of sister chromatid exchanges (Latt, 1974). Hoehn and Martin (1972) have demonstrated that the addition of Mitomycin $C$ to human fibroblast cultures produces instability in the $\mathrm{C}$-band sizes with some sister chromatids possessing differing amounts of $\mathrm{C}$-band heterochromatin. Following cessation of Mitomycin $C$ treatment these variants have been shown to be distributed to succeeding generations in a clonal fashion (Hoehn and Martin, 1973). Their data suggest that unequal sister chromatid interchanges may be the origin of $C$-band variants. If one considers a duplication of a block of heterochromatin containing more than one identifiable region it seems apparent that an unequal sister chromatid exchange would produce two blocks of identical patterns. Although much of the data contained in this study are consistent with such a mechanism, there are examples presented which clearly are not compatible with this model. For example, the large C-band on the chromosome 1 which contains two blocks of Giemsa-11 heterochromatin of differing sizes (Figure 8) and the chromosome 9 with two different sizes (Figure 12).

Gene Amplification. A brief discussion of gene amplification and its possible relation to changes in heterochromatin is included here because there appear to be many similarities due to the repetitive nature of ampl ified genes.

Individuals who have been treated with the anti-folate drug metho- 
trexate have been shown to contain an increased activity of dihydrofolate reductase, the target enzyme for methotrexate (Bertino et. al., 1963). Cells selected for methotrexate resistance in culture have been able to grow in concentrations in excess of 10,000 times the concentration that kills normal sensitive cells (Bostock et. al., 1980). Resistant murine cells have been reported to contain an unusually large amount of messenger RNA for dihyrofolate reductase (Chang and Littlefield, 1976) and also an increase in the number of gene copies (Alt et. a1., 1978). Resistant cells contain large marker chromosomes which contain distinctive homogeneously staining regions (HSRs) by the G-banding technique (Biedler and Spengler, 1976) and have been shown to hybridize labeled messenger RNA for dihydrofolate reductase indicating these genes to be present within the HSRs (Nunberg et. a1., 1978). The latter group reports that the repeat length for the amplified segments were of the order of $10^{6}$ base pairs as compared to 1,600 b.p. for dihydrofolate reductase mRNA (Chang et. al., 1978) or 40,000 b.p. for the length of genomic DNA coding for mRNA and intervenịng sequences (Schimke et. al., 1979). Obviously there is a huge amount of DNA contained in these HSRs which is independent of the actual gene selected for. Bostock and Clark (1980) have reported a resistant mouse cell line containing HSRs which are composed of many fine C-bands closely linked together. Staining of these HSRs with the AT specific flourochrome 33258 Hoechst reveals them to be similar to the centromeric heterochromatin in that they are exceedingly rich in AT base pairs. Hybridization in situ with ${ }^{125}$ nick translated mouse satellite DNA shows the HSRs to contain large amounts of sequence that will cross hybridize under fairly stringent conditions. They also performed total 
satellite DNA analyses of resistant and sensitive cell nucleii by analytical density gradients and found that the mean content of satellite DNA of density $1.691 \mathrm{~g} \mathrm{~cm}^{-3}$ was $15.62 \%$ for the resistant cells and $9.26 \%$ for sensitive cells without HSRs, an increase of about $60 \%$. They calculated the repeat length to be on the order of $3 \times 10^{6}$ b.p. in length, of which about two thirds ( $2 \times 10^{6}$ b.p.) must be satellite DNA. Miller et. al. (1979) have reported rat hepatoma cell lines containing chromosomes with what they term differentially staining regions (DRSs) in the locations which normally contain the genes coding for 185 and 285 ribosomal RNA (nucleolar organizing regions). These DSRs stain mildly with C-banding and although they contain a 10 fold increase in ribosomal genes there is more DNA amplified than can be accounted for by these genes alone. This extra DNA probably reflects sequences which are adjacent to the actual structural gene and may be responsible for the amplification process. These repetitive sequences must therefore serve a major function in gene amplification which is not yet clearly understood. Repetitive DNA is not restricted to centromeric locations in its chromosomal distributions but has also been shown to exist in interstitial chromatin (Gosden et. al., 1975a). Perhaps most or all genes are surrounded by sequences which can be recognized by site specific endonucleases and amplified to such great extents. Even if the amplification sequences were randomly chosen it would not take long for a resistant cell to out-compete other cells in a culture and pass on this resistance to daughter cells. It may be a normal function involved with such topics as cellular evolution as these site specific endonucleases have been isolated in testis cells of the African green monkey Cerco- 
pithecus aethiops (Brown et. a1., 1978).

I add this discussion of gene amplification because it affords a contrast to the other proposed mechanisms of chromosome variation and in many ways is compatible with some of the more graded ranges in sizes of heterochromatin such as those found in G-band positive heterochromatin. These variations might represent changes on a fine subcellular level as opposed to large quantum changes in Giemsa-11 positive heterochromatin which might reflect changes on a gross cytological level. 


\section{REFERENCES}

Alt, F.W., Kellems, R.E., Bertino, J.R. and Schimke, R.T.: Selective multiplication of dihydrofolate reductase genes in methotrexateresistant variants of cultured murine cells. J. Biol. Chem. 253:1357-1370 (1978)

Angell, R.R. and Jacobs, P.A.: Lateral asymmetry in human constitutive heterochromatin. Chromosoma 51:301-310 (1975)

Arrighi, F.E. and Hsu, T.C.: Localization of heterochromatin in human chromosomes. Cytogenetics 10:81-86 (1971)

Bahr, G.F., Mikel, U. and Engler, W.F.: Correlates of chromosomal banding at the level of ultrastructure. Chromosome Identification Techniques and Applications in Biology and Medicine (T. Caspersson and L. Zeck, eds.) 280-289, Academic Press, N.Y. (1973)

Barr, M.L. and Bertram, E.G.: A morphological distinction between neurones of the male and female and the behavior of the nuclear satellite during accelerated nucleoprotein synthesis. Nature (Lond.) 163:676-677 (1949)

Beidler, J.L. and Spengler, B.A.: Metaphase chromosome anomaly: association with drug resistance and cell specific products. Science 191: 185-187 (1976)

Bertino, J.R., Donahue, D.M., Simmons, B., Gabrio, B.W., Silbar, R. and Huennekens, F.M.: The "induction" of dihydrofolate reductase activity in leukocytes and erythrocytes in patients treated with amethopterin. J. Clin. Invest. 42:466-475 (1963)

Bobrow, M., Madan, K. and Pearson, P.L.: Staining of some specific regions of human chromosomes, particularly the secondary constriction of no. 9. Nature New Biol. 238:122-124 (1972)

Bostock, C.J. and Clark, E.M.: Satellite DNA in large marker chromosomes of methotrexate-resistant mouse cells. Cel1 19:709-715 (1980)

Brown, F.L., Musich, P.R. and Maio, J.J.: Cae I: an endonuclease isolated from the African green monkey kidney with properties indicating site-specific cleavage of homologous and heterologous mammalian DNA. Nucl. Acids Res. $\underline{5}: 1093-1107$ (1978)

Brown, S.W.: Heterochromatin. Science 151:417-425 (1966) 
Bühler, E.M., Tsuchimoto, T., Jurik, L.P. and Stalder, G.R.: Satellite DNA III and alkaline giemsa staining. Humangenetik 26:329-333 (1975)

Chang, A.C.Y., Nunberg, J.H., Kaufman, R.J., Erlich, H.A., Schimke, R.T. and Cohen, S.N.: Phenotypic expression in E. coli of a DNA sequence coding for mouse dihydrofolate reductase. Nature 275:617-624 (1978)

Chang, S.E. and Littlefield, J.W.: Elevated dihydrofolate reductase messenger RNA levels in methotrexate-resistant BHK cells. Cell 7: 391-396 (1976)

Chuang, C.R.: The genetic constitution of human nucleoli. J. Cell Biol. 63:62a (1974)

Chuang, C.R. and Saunders, G.F.: Complexity of human satellite A DNA. Biochem. Biophys. Res. Commun. 57:1221-1230 (1974)

Comings, D.E.: Mechanisms of chromosome banding and implications for chromosome structure. Ann. Rev. Genet. 12:25-46 (1978)

Comings, D.E., Avelino, E., Okada, T. and Wyandt, H.E.: The mechanism of C- and G-banding of chromosomes. Exp. Cell Res. 77:469-493 (1973)

Comings, D.E., and Avelino, E.: Mechanisms of chromosome banding. VII. Interaction of methylene blue with DNA and chromatin. Chromosoma 51:365-381 (1975)

Comings, D.E. and Okada, T.A.: Nuclear proteins. III. The fibrillar nature of the nuclear matrix. Exp. Cell Res. 103:341-360 (1976)

Corneo, G., Ginelli, E. and Polli, E.: Repeated sequences in human DNA. J. Molec. Biol. 48:319-327 (1970)

Craig-Holmes, A.P., Moore, F.B. and Shaw, M.W.: Polymorphism of human C-band heterochromatin. II. Family studies with suggestive evidence for somatic crossing over. Am. J. Hum. Genet. 27: 178-189 (1973)

Eiberg, H.: New selective giemsa technique for human chromosomes. Cd staining. Nature 248:55 (1974)

Evans, H.J.: Some facts and fancies relating to chromosome structure in man. Adv. Hum. Genet. $8: 347-438$ (1976)

Gagne, R. and Laberge, C.: Specific cytological recognition of the heterochromatic segment of number 9 chromosome in man. Exp. Cel1 Res. 73:239-242 (1972)

Galloway, S.M. and Evans, H.J.: Asymmetrical C-bands and satellite DNA in man. Exp. Cell Res. 94:454-456 (1975) 
Geraedts, J.P.M., Pearson, P.L., Van der Ploeg, M. and Vossepoel, A.M.: Polymorphisms for human chromosomes 1 and $Y$. Feulgen and $u . v$. DNA measurements. Exp. Ce11 Res. 95:9-14 (1975)

Gosden, J.R., Buckland, R.A., Clayton, R.P. and Evans, H.J.: The location of condensed and dispersed chromatin. Exp. Cell Res. 92:138-147 (1975a)

Gosden, J.R., Mitchel1, A.R., Buckland, R.A., Clayton, R.P. and Evans, H.J.: The localization of four human satellite DNAs on human chromosomes. Exp. Cell Res. 92:148-158 (1975b)

Heitz, E.: Das heterochromatin der moose. I. Jahrb. Wiss. Bot. 69: 762-818 (1928)

Hoehn, H. and Martin, G.M.: Heritable alteration of human constitutive heterochromatin induced by Mitomycin C. Exp. Cell Res. 75:275278 (1972)

Hoehn, H. and Martin, G.M.: Clonal variants of constitutive heterochromatin of human fibroblasts after recovery from Mitomycin C treatment. Chromosoma 43:203-210 (1973)

Holmquist, G.: The mechanism of C-banding: depurination and $\beta$-elimination.

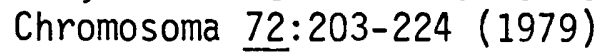

Hungerford, D.A., Ashton, F.T., Balaban, G.B., LaBadie, G.U., Messatzzia, L.R., Haller, G. and Miller, A.E.: The C-group pachytene bivalent with a locus characteristic for parachromosomally situated particulate bodies (parameres): A provisional map in human males. Proc. Nat. Acad. Sci. (USA) 69:2165-2168 (1972)

John, B.: Myths and mechanisms of meiosis. Chromosoma 54:295-325 (1976)

Jones, K.W.: Molecular structure of human chromosomes. (J.J.Yunis, ed.) p. 323, Academic Press, N.Y. (1977)

Jones, K.W. and Corneo, G.: Location of satellite and homogeneous DNA sequences on human chromosomes. Nature (Lond.) New Biol. 233: 268-271 (1971)

Jones, K.W., Prosser, J., Corneo, G., and Ginelli, E.: The chromosomal location of human satellite DNA III. Chromosoma 42:445-451 (1973)

Jones, K.W., Purdom, I.F., Prosser, J. and Corneo, G.: The chromosomal localization of human satellite DNA I. Chromosoma 49:161-171 (1974)

Kurnit, D.M.: Satellite DNA and heterochromatin variants: The case for unequal mitotic crossing over. Hum Genet. 47:169-186 (1979) 
Latt, S.A.: Sister chromatid exchanges, indices of human chromosome damage and repair: Detection by fluorescence and induction by Mitomycin C. Proc. Nat. Acad. Sci. (USA) 71:3162-3166 (1974)

Latt, S.A., Davidson, R.C., Lin, M.S. and Gerald, P.S.: Lateral asymmetry in the fluorescence of human $Y$ chromosomes stained with 33258 Hoechst. Exp. Cel1 Res. 87:425-429 (1974)

Lin, M.S., Latt, S.A. and Davidson, R.C.: Microfluorometric detection of asymmetry in the centromeric region of mouse chromosomes. Exp. Cel1 Res. 86:392-395 (1974)

Lin. M.S. and $A l f i$, O.S.: Detection of lateral asymmetry in the C-band of human chromosomes by BrdU-DAPI fluorescence. Somat. Ce11 Genet. 4:603-608 (1978)

Lyons, M.F.: Gene action in the X-chromosome of the mouse. Nature (Lond.) 190:372-373 (1961)

Macaya, G., Thiery, J. and Bernardi, G.: DNA sequences in man. Molecular Structure of Human Chromosomes. $35-38$ (J. Yunis, ed.) Academic Press, N.Y. (1977)

Madan, K.: An extra band in human 9qh+ chromosomes. Hum. Genet. 43: 259-264 (1978)

Madan, K. and Bruinsma, A.H.: C-band polymorphism in human chromosome 6 . C1 in. Genet. 15:193-197 (1979)

Marks, G.E.: The nature of centromeric dots in Nigella chromosomes. Chromosoma 62:369-373 (1977)

Marsden, M.P.F. and Laemmli, U.K.: Metaphase chromosome structure: evidence for a radial loop model. Cell 17:849-858 (1979)

Marx, K.A., Allen, J.R. and Hearst, J.E.: Characterization of the repetitious human DNA families. Biochim. et. Biophys. Acta 425:129-147 (1976)

Matsukuma, S.A. and Utakoji, T.: Uneven extraction of protein in Chinese hamster chromosomes during G-staining procedures. Exp. Ce11 Res. 97:297-303 (1976)

McKay, R.D.G.: The mechanism of $G$ and $C$ banding in mammalian metaphase chromosomes. Chromosoma 44:1-14 (1973)

Merrick, S., Ledley, R.S. and Lubs, H.A.: Production of $G$ and $C$ banding with progressive trypsin treatment. Pediatr. Res. $7: 39-44$ (1973)

Miller, 0.J., Tantravahi, R., Miller, D.A., Yu, L., Szabo, P. and Prensky, W.: Marked increase in ribosomal RNA gene multiplicity in a rat hepatoma cell 1ine. Chromosoma 71:183-195 (1979) 
Mittermayer, C., Madreiter, H., Lederer, B. and Sandritter, S.:

Differential acid hydrolys is of euchromatin and heterochromatin

(Biochemical, histochemical and morphological studies). Beitr.

Pathol. 143:157-171 (1971)

Müller, H.J., Klinger, H.P. and Glasser, M.: Chromosome polymorphism in a human newborn population. II. Potentials of polymorphic chromosome variants for characterizing the idiogram of an individual. Cytogenet. Cel1 Genet. 15:239-256 (1975)

Nunberg, J.H., Kaufman, R.J., Schimke, R.T., Urlaub, G. and Chas in, L.A.: Amplified dihydrofolate reductase genes are localized to a homogeneously staining region of a single chromosome in a methotrexate-resistant Chinese hamster ovary cell line. Proc. Nat. Acad. Sci. (USA) 75:5553-5556 (1978)

Okada, T.A. and Comings, D.E.: Mechanisms of chromosome banding. III. Similarity between G-bands of mitotic chromosomes and chromomeres of meiotic chromosomes. Chromosoma 48:65-71 (1974)

Okada, T.A. and Comings, D.E.: Higher order structure of chromosomes. Chromosoma 72:1-14 (1979)

Page, B.M.: Identification of chromosome 9 in human male meiosis. Cytogenet. Cell Genet. 12:254-263 (1973)

Paulson, J.R. and Laemmli, U.K.: The structure of histone-depleted metaphase chromosomes. Cel1 12:817-828 (1977)

Rodman, T.C.: Human chromosome banding by Feulgen stain aids in localizing classes of chromatin. Science 184:171-173 (1974)

Saunders, G.F., Hsu, T.C., Getz, M.J., Simes, E.L. and Arrighi, F.E.: Locations of a human satellite DNA in human chromosomes. Nature New Biol. (Lond.) 236:244-246 (1972)

Saunders, G.F., Chuang, C.R. and Sawada, H.: Genome complexity and in vitro transcription in human leukemic leucocytes.

Acta Haematol. 54:227-233 (1975)

Schimke, R.T., Brown, P.C., Kaufman, R.J. and Nunberg, J.H.:

Structure and localization of dihydrofolate reductase genes in methotrexate-resistant cultured cells. ICN-UCLA Symposium on Eukaryote Gene Expression, in press (1980)

Seabright, M.: A rapid banding technique for human chromosomes. Lancet 2: 971-972 (1971)

Wyandt, H.E., Wysham, D.G., Minden, S.K., Anderson, R.S. and Hecht, F.: Mechanisms of giemsa banding of chromosomes. I. Giemsa-11 banding with azure and eosin. Exp. Cell Res. 102:85-94 (1976) 
Yunis, J.J.: High resolution of human chromosomes. Science 191:

$1268-1270$ (1976) 